\title{
Catalytic Enantioselective Aza-Henry Reaction with Broad Substrate Scope
}

\author{
Claudio Palomo, ${ }^{\star}$ Mikel Oiarbide, Antonio Laso, Rosa López \\ Departamento de Química Orgánica I, Facultad de Química, Universidad del País Vasco, Apdo. \\ 1072, 20080 San Sebastián, Spain.
}

\section{SUPPORTING INFORMATION}

\section{INDEX}

A) General information...................................................... 1

B) General procedure for the aza-Henry reaction of nitromethane and nitroethane with $\alpha$-amido sulfones under PTC conditions................... 2

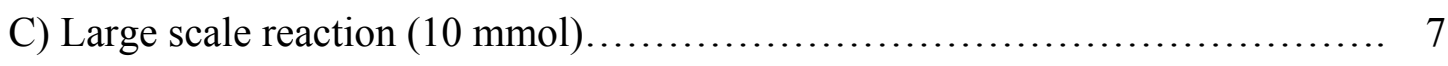

D) Assignment of the absolute configuration of adducts..................... 8

E) ${ }^{1} \mathrm{H}$ and ${ }^{13} \mathrm{C}$ NMR data of selected compounds............................ 9

F) HPLC chromatograms of selected products............................. 17

\section{A) General information:}

All reactions were carried out with efficient magnetic stirring. Toluene was dried in the presence of sodium metal. Nitromethane and nitroethane were reagent grade and used as received. Purchased cesium hydroxide monohydrate was kept in dessicator before use. $\mathrm{N}$-Benzylquininium chloride $5, \mathrm{~N}$-benzyl cinchonidinium chloride 6, and $\mathrm{N}$ benzyl cinchoninium chloride 7 were used as received. Quaternary ammonium compounds 8 and $\mathbf{9}$ were prepared according to literature procedures. ${ }^{1} \alpha$-Amidosulfones 1a-n were prepared according to described procedures. ${ }^{2}$ Purification of reaction products was carried out by flash column chromatography using silica gel 60 (0.040$0.063 \mathrm{~mm}, 230-400 \mathrm{mesh}$ ). Analytical thin layer chromatography (TLC) was performed on $0.25 \mathrm{~mm}$ silica gel 60 -F plates. Visualization was accomplished with UV light and a solution obtained by admixing in $470 \mathrm{~mL}$ of water ammonium molybdate $(21 \mathrm{~g})$, cerium sulphate $(1 \mathrm{~g})$ and concentrated sulphuric acid $(31 \mathrm{~mL})$, followed by heating. Melting points were measured with a Buchi SMP-20 melting point apparatus and are uncorrected. Infrared spectra were recorded on a Nicolet Avatar 360 FT-IR spectrometer. ${ }^{1} \mathrm{H}$ and ${ }^{13} \mathrm{C}$ NMR spectra were recorded on Varian Gemini 200, Bruker

\footnotetext{
${ }^{1}$ Martin J. O'Donnell, Shengde Wu and John C. Huffman, Tetrahedron,1994, 50, 4507-4518.

2 a) Pearson, W.H.; Lindbeck, A.C.; Kampf, J.W. J. Am. Chem. Soc.,1993,115, 2622. b) Mecozzi, T.; Petrini, M. J. Org. Chem.1999,64, 8970-8972.
} 
Advance-DPX-300, and Bruker Advance-500 and are reported in ppm from internal tetramethylsilane (TMS). Analytical high performance liquid chromatography (HPLC) was performed on waters-600E and Hewlett Packard series 1050 chromatographs, equipped with diode array UV detector, using Daicel Chiralpak AD, IA, AS, and Chiralcel OD, OJ columns. Elemental analyses were performed with a Leco CHNS-932. Optical rotations were recorded on a Perkin Elmer polarimeter. Racemic $\beta$-nitroamines were prepared according to the procedure of Petrini. ${ }^{3}$

\section{B) General procedure for the aza-Henry reaction of nitromethane and nitroethane with $\alpha$-amido sulfones under PTC conditions.}

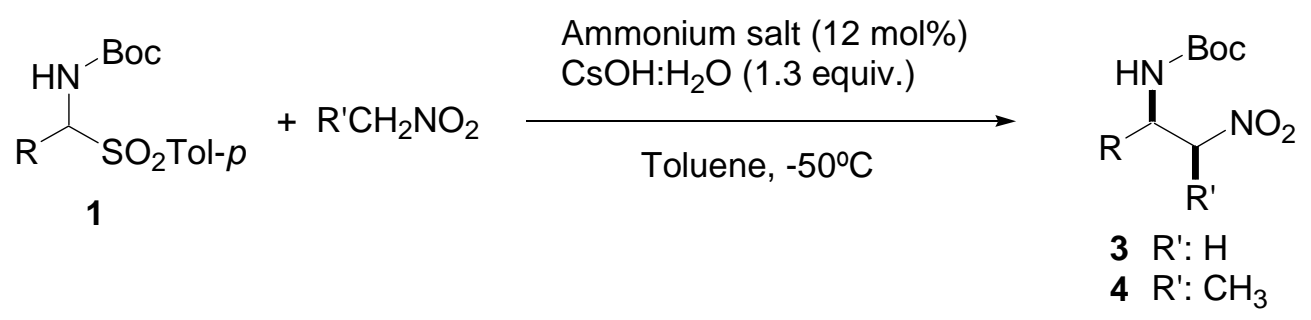

To a mixture of the corresponding $\alpha$-amidosulfone $1(0.5 \mathrm{mmol})$ and N-benzyl quininium chloride 5 ( $27 \mathrm{mg}, 0.06 \mathrm{mmol}$ ) (or the corresponding salt 6-9) in toluene (1.5 $\mathrm{mL})$ were successively added $\mathrm{CH}_{3} \mathrm{NO}_{2}(0.13 \mathrm{~mL}, 2.5 \mathrm{mmol})$ and, after cooling to -50 ${ }^{\circ} \mathrm{C}, \mathrm{CsOH} \cdot \mathrm{H}_{2} \mathrm{O}(101 \mathrm{mg}, 0.65 \mathrm{mmol})$. The reaction mixture was stirred under a nitrogen atmosphere at the same temperature for 40 hours. The mixture was then quenched with $\mathrm{HCl}(1.5 \mathrm{~mL}, 0.1 \mathrm{~N})$ and extracted with $\mathrm{CH}_{2} \mathrm{Cl}_{2}(3 \times 2 \mathrm{~mL})$. The organic layer was washed with $\mathrm{HCl}(1 \times 2 \mathrm{~mL}, 0.1 \mathrm{~N})$, dried over $\mathrm{MgSO}_{4}$, and concentrated under reduced pressure. The crude product was purified by flash column chromatography using mixtures of ethyl acetate/hexane as the eluant.

The same procedure was employed for the reactions using nitroethane instead of nitromethane.

Note: Whereas the use of less than 1.3 equivalents of $\mathrm{CsOH} \cdot \mathrm{H}_{2} \mathrm{O}$ may lead to inferior results, the use of larger quantities of $\mathrm{CsOH} \cdot \mathrm{H}_{2} \mathrm{O}$ (checked up to 2.0 mol equivalents) had no detrimental effect.

\section{(R)-1-Nitro-4-phenylbutan-2-ylcarbamic acid tert-butyl ester (3a)}

The title compound was prepared according to the general procedure starting from $\alpha$ -

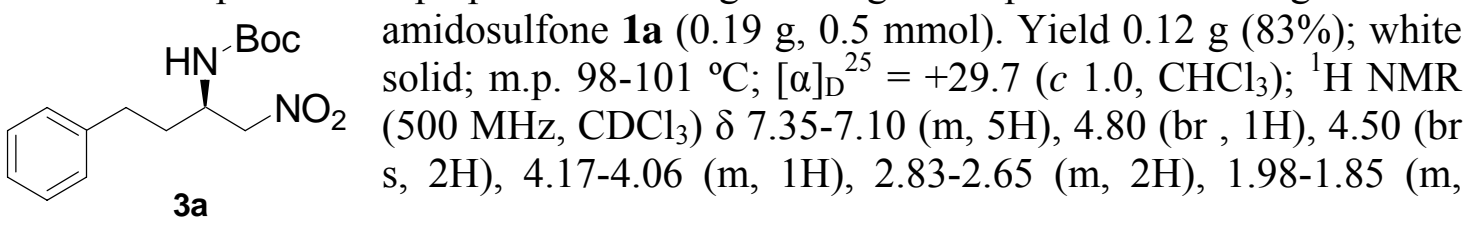

\footnotetext{
${ }^{3}$ Ballini, R.; Petrini, M. Tetrahedron Lett.1999,40, 4449-4452
} 
2H), $1.40(\mathrm{~s}, 9 \mathrm{H}) ;{ }^{13} \mathrm{C}$ NMR $\left(125 \mathrm{MHz}, \mathrm{CDCl}_{3}\right) \delta 155.4,140.7,129.0,128.7,126.7$, 80.7, 78.7, 49.3, 33.8, 32.6, 28.7; Chiral HPLC (chiralpak AD column; hexane:iPrOH 98:2;1.0 mL/min, $210 \mathrm{~nm}$ ) $\mathrm{Rt}_{\text {major }}=30 \mathrm{~min}, \mathrm{Rt}_{\text {minor }}=34.5 \mathrm{~min}, 96 \%$ ee; Anal. calcd. for $\mathrm{C}_{15} \mathrm{H}_{22} \mathrm{~N}_{2} \mathrm{O}_{4}$ (294.35): C, 61.21; H, 7.53; N, 3.52. Found: C, 61.72; H, 7.60; N, 3.49.

\section{(R)-1-Nitrobutan-2-ylcarbamic acid tert-butyl ester (3b)}

The title compound was prepared according to the general procedure starting from $\alpha$ -<smiles>CCC(CNC(=O)OCc1ccccc1)NC(=O)O[Na]</smiles>

$3 \mathbf{b}$ amido sulfone $1 \mathrm{~b}(0.15 \mathrm{~g}, 0.5 \mathrm{mmol})$. Yield $87.3 \mathrm{mg}(80 \%)$; white solid; m.p. $74-76{ }^{\circ} \mathrm{C} ;[\alpha]_{\mathrm{D}}{ }^{25}=+51.2\left(\right.$ c 1.0, $\left.\mathrm{CHCl}_{3}\right) ;{ }^{1} \mathrm{H}$ NMR $(500$ $\left.\mathrm{MHz}, \mathrm{CDCl}_{3}\right) \delta 4.80(1 \mathrm{H}, \mathrm{br}), 4.54(2 \mathrm{H}, \mathrm{br}), 4.04(1 \mathrm{H}, \mathrm{m}), 1.64-1.54$ $(\mathrm{m}, 2 \mathrm{H}), 1.46(\mathrm{~s}, 9 \mathrm{H}), 1.02(3 \mathrm{H}, \mathrm{t}, J=7.4 \mathrm{~Hz}) ;{ }^{13} \mathrm{C}$ NMR $(125 \mathrm{MHz}$, $\left.\mathrm{CDCl}_{3}\right) \delta 155.2,80.3,78.1,50.8,28.3,25.1,10,5$; Chiral HPLC (chiralpak AD column; hexane:iPrOH 95:5; $1.0 \mathrm{~mL} / \mathrm{min}, 254 \mathrm{~nm}$ ) $\mathrm{Rt}_{\text {major }}=10.0 \mathrm{~min}$, $\mathrm{Rt}_{\text {minor }}=12.5 \mathrm{~min}, 96 \%$ ee; Anal. calcd. for $\mathrm{C}_{9} \mathrm{H}_{18} \mathrm{~N}_{2} \mathrm{O}_{4}$ (218.25): C, 49.53; H, 8.31; N, 12.84. Found: C, 49.28; H, 8.42; N, 12.68 .

\section{(2R)-1-Nitropentan-2-ylcarbamic acid tert-butyl ester (3c).}

The title compound was prepared according to the general procedure starting from $\alpha$ -

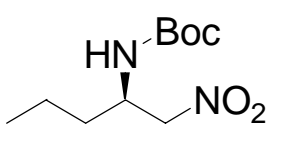
amido sulfone 1c $(0.16 \mathrm{~g}, 0.5 \mathrm{mmol})$. Yield $90.6 \mathrm{mg}(78 \%)$; white solid; m.p. $101-103{ }^{\circ} \mathrm{C}$; $[\alpha]_{\mathrm{D}}{ }^{25}=+44.2\left(\right.$ c $\left.1.0, \mathrm{CHCl}_{3}\right) ;{ }^{1} \mathrm{H}$ NMR $(500$ $\left.\mathrm{MHz}, \mathrm{CDCl}_{3}\right) \delta 4.80(1 \mathrm{H}, \mathrm{br}), 4.53(2 \mathrm{H}, \mathrm{br}), 4.11(1 \mathrm{H}, \mathrm{m}), 1.56(2 \mathrm{H}$, m), $1.46(9 \mathrm{H}, \mathrm{s}), 1.43(2 \mathrm{H}, \mathrm{m}), 0.97(3 \mathrm{H}, \mathrm{t}, J=7.3 \mathrm{~Hz}) ;{ }^{13} \mathrm{C}$ NMR $\left(125 \mathrm{MHz} \mathrm{CDCl}_{3}\right) \delta 155.0,80.1,78.4,49.0,33.9,28.3,19.1,13.6$;

Chiral HPLC (chiralpak AD column; hexane:iPrOH 90:10;0.8 mL/min, $210 \mathrm{~nm}$ ) $\mathrm{Rt}_{\text {major }}=8.5 \mathrm{~min}, \mathrm{Rt}_{\text {minor }}=9.5 \mathrm{~min}, 94 \%$ ee; Anal. calcd. for $\mathrm{C}_{10} \mathrm{H}_{20} \mathrm{~N}_{2} \mathrm{O}_{4}$ (232.28): $\mathrm{C}$, 51.71; H, 8.68; N, 12.06. Found: C, 51.52; H, 8.71; N, 12.51.

\section{(2R)-1-Nitrooct-2-ylcarbamic acid tert-butyl ester (3d).}

The title compound was prepared according to the general procedure starting from $\alpha$ -

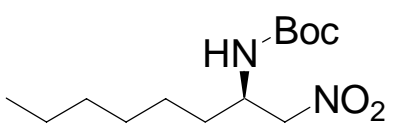
amidosulfone 1d (0.18 g, $0.5 \mathrm{mmol})$. Yield $107 \mathrm{mg}(78 \%)$; white solid; m.p. $118-120{ }^{\circ} \mathrm{C} ;[\alpha]_{\mathrm{D}}{ }^{25}=+27.3\left(\right.$ c $\left.1.0, \mathrm{CHCl}_{3}\right)$; ${ }^{1} \mathrm{H}$ NMR $\left(500 \mathrm{MHz}, \mathrm{CDCl}_{3}\right) \delta 4.78(1 \mathrm{H}, \mathrm{br}), 4.52(2 \mathrm{H}, \mathrm{br})$, 3d $4.08(1 \mathrm{H}, \mathrm{m}), 1.55(2 \mathrm{H}, \mathrm{m}), 1.50-1.46(4 \mathrm{H}, \mathrm{m}), 1.45(9 \mathrm{H}, \mathrm{s})$, 1.44-1.40 $(2 \mathrm{H}, \mathrm{m}), 0.95(3 \mathrm{H}, \mathrm{t}, J=5.6 \mathrm{~Hz}) ;{ }^{13} \mathrm{C}$ NMR $(125$

$\left.\mathrm{MHz}, \mathrm{CDCl}_{3}\right) \delta 155.1,80.2,78.4,49.2,31.5,28.7,28.2,27.9,25.8,22.5,13.9$; Chiral HPLC (chiralpak AD column; hexane: $\mathrm{PrOH} 95: 5 ; 0.5 \mathrm{~mL} / \mathrm{min}, 210 \mathrm{~nm}$ ) $\mathrm{Rt}_{\text {major }}=8.5$ min, $\mathrm{Rt}_{\text {minor }}=9.0 \mathrm{~min}, 98 \%$ ee; Anal. calcd. for $\mathrm{C}_{13} \mathrm{H}_{26} \mathrm{~N}_{2} \mathrm{O}_{4}$ (274.36): C, 56.91; $\mathrm{H}, 9.55$; N, 10.21. Found: C, 56.62; H, 9.61; N, 10.53.

\section{(2R)-4-Methyl-1-nitropent-2-ylcarbamic acid tert-butyl ester (3e)}

The title compound was prepared according to the general procedure starting from $\alpha$ -

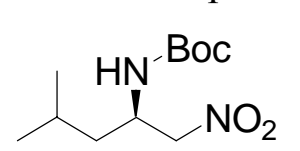

$3 e$ amido sulfone 1e $(0.17 \mathrm{~g}, 0.5 \mathrm{mmol})$. Yield $97.3 \mathrm{mg}(79 \%)$; white solid; m.p. $110-113{ }^{\circ} \mathrm{C} ;[\alpha]_{\mathrm{D}}{ }^{25}=+26.4\left(\right.$ c $\left.1.0, \mathrm{CHCl}_{3}\right) ;{ }^{1} \mathrm{H}$ NMR $\left(500 \mathrm{MHz}, \mathrm{CDCl}_{3}\right) \delta 4.82(1 \mathrm{H}$, br) $4.56(2 \mathrm{H}$, br $), 4.14(1 \mathrm{H}, \mathrm{m})$, $1.95-1.85(1 \mathrm{H}, \mathrm{m}), 1.62(2 \mathrm{H}, \mathrm{m}), 1.46(9 \mathrm{H}, \mathrm{s}), 0.96(6 \mathrm{H}, \mathrm{dd}, J=6.3$

$\mathrm{Hz}, J=13.3 \mathrm{~Hz}) ;{ }^{13} \mathrm{C}$ NMR $\left(125 \mathrm{MHz}, \mathrm{CDCl}_{3}\right) \delta 153.7,80.8,78.5$,

48.7, 39.3 28.0, 24.8, 21.7; Chiral HPLC (chiralpak AD column; hexane:iPrOH 90:10; $0.8 \mathrm{~mL} / \mathrm{min}, 210 \mathrm{~nm}) \mathrm{Rt}_{\text {major }}=8.5 \mathrm{~min}, \mathrm{Rt}_{\text {minor }}=9.5 \mathrm{~min}, 97 \%$ ee; Anal. calcd. for $\mathrm{C}_{11} \mathrm{H}_{22} \mathrm{~N}_{2} \mathrm{O}_{4}$ (266.29): C, 56.64; H, 9.00; N, 11.37. Found: C, 56.62; H, 9.11; N, 11.53. 
(2R)-3-Methyl-1-nitrobut-2-ylcarbamic acid tert-butyl ester (3f)

The title compound was prepared according to the general procedure starting from $\alpha$ -<smiles>CC(C)C(C[N+](=O)[O-])NC(=O)OC(C)(C)C</smiles>

$3 f$ amido sulfone 1f $(0.16 \mathrm{~g}, 0.5 \mathrm{mmol})$. Yield $94.1 \mathrm{mg}(81 \%)$; white solid; m.p. $80-83{ }^{\circ} \mathrm{C}$; $[\alpha]_{\mathrm{D}}{ }^{25}=+34.8\left(\right.$ c $\left.1.0, \mathrm{CH}_{2} \mathrm{Cl}_{2}\right) ;{ }^{1} \mathrm{H}$ NMR $(500 \mathrm{MHz}$, $\left.\mathrm{CDCl}_{3}\right) \delta 4.85(1 \mathrm{H}, \mathrm{br}), 4.57(1 \mathrm{H}, \mathrm{m}), 4.55(1 \mathrm{H}, \mathrm{m}), 4.10-3.95(1 \mathrm{H}, \mathrm{m})$, $1.90(1 \mathrm{H}, \mathrm{m}),, 1.45(9 \mathrm{H}, \mathrm{s}), 1.01(6 \mathrm{H}, \mathrm{dd}, J=6.7,9.5 \mathrm{~Hz}) ;{ }^{13} \mathrm{C}$ NMR $\left(125 \mathrm{MHz}, \mathrm{CDCl}_{3}\right) \delta$ 155.2, 80.1, 74.4, 54.7, 30.1, 28.2, 19.4, 18.5;

Chiral HPLC (chiralpak AD column; hexane:iPrOH 90:10; $0.8 \mathrm{~mL} / \mathrm{min}, 210 \mathrm{~nm}$ ) $\mathrm{Rt}_{\text {major }}=9.0 \mathrm{~min}, \mathrm{Rt}_{\text {minor }}=10.0 \mathrm{~min}, 95 \%$ ee; Anal. calcd. for $\mathrm{C}_{10} \mathrm{H}_{22} \mathrm{~N}_{2} \mathrm{O}_{4}$ (232.28): $\mathrm{C}$, 51.71; H, 8.68; N, 12.06. Found: C, 51.42; H, 8.41; N, 12.13.

\section{(2R)-2-Cyclohexyl-1-nitroeth-2-ylcarbamic acid tert-butyl ester (3g)}

The title compound was prepared according to the general procedure starting from $\alpha$ -

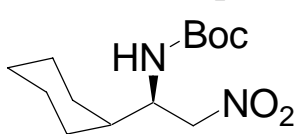

$3 g$ amido sulfone $1 \mathrm{~g}(0.18 \mathrm{~g}, 0.5 \mathrm{mmol})$. Yield $104 \mathrm{mg}$ (77\%); white solid; m.p. $128-130{ }^{\circ} \mathrm{C} ;[\alpha]_{\mathrm{D}}{ }^{25}=+24\left(\right.$ c 1.0, $\left.\mathrm{CHCl}_{3}\right) ;{ }^{1} \mathrm{H}$ NMR $(500$ $\left.\mathrm{MHz}, \mathrm{CDCl}_{3}\right) \delta 4.83(1 \mathrm{H}, \mathrm{br}), 4.50(1 \mathrm{H}, \mathrm{br}), 4.48(1 \mathrm{H}, \mathrm{br}), 4.00-$ $3.85(1 \mathrm{H}, \mathrm{m}), 1.90-1.42(5 \mathrm{H}, \mathrm{m}), 1.40(9 \mathrm{H}, \mathrm{s}), 1.30-0.97(6 \mathrm{H}, \mathrm{m})$; ${ }^{13} \mathrm{C}$ NMR $\left(125 \mathrm{MHz}, \mathrm{CDCl}_{3}\right) \delta 155.8,80.3,76.3,53.6,39.2,29.7$,

28.9, 28.3, 25.7, 25.5; Chiral HPLC (chiralpak AD column; hexane:iPrOH 90:10; 1.0 $\mathrm{mL} / \mathrm{min}, 210 \mathrm{~nm}) \mathrm{Rt}_{\text {major }}=8.0 \mathrm{~min}, \mathrm{Rt}_{\text {minor }}=9.0 \mathrm{~min}, 98 \%$ ee; Anal. calcd. for $\mathrm{C}_{13} \mathrm{H}_{24} \mathrm{~N}_{2} \mathrm{O}_{4}$ (272.34): C, 57.33; H, 8.88; N, 10.29. Found: C, 57.21; H, 8.92; N, 10.83.

(2R)-1-Nitro-2-phenyleth-2-ylcarbamic acid tert-butyl ester (3h)

The title compound was prepared according to the general procedure starting from<smiles>CC(C)(C)OC(=O)N[C@H](C[N+](=O)[O-])c1ccccc1</smiles>

3h amido sulfone 1a $(0.10 \mathrm{~g}, 0.5 \mathrm{mmol})$. Yield $0.10 \mathrm{~g}(79 \%)$; white solid; m.p. $105-106^{\circ} \mathrm{C}$ (Lit. ${ }^{4}: \mathrm{mp} 116-118^{\circ} \mathrm{C}, 60 \%$ ee ); $[\alpha]_{\mathrm{D}}{ }^{25}=-20.1$ (c 1.0, $\left.\mathrm{CHCl}_{3}\right)\left[\right.$ Lit. $^{5}[\alpha]_{\mathrm{D}}{ }^{25}=-26.5$ (c 1.0, acetone); ${ }^{1} \mathrm{H}$ NMR (500 $\left.\mathrm{MHz} \mathrm{CDCl}_{3}\right) \delta 7.39(1 \mathrm{H}, \mathrm{d}, J=7.0 \mathrm{~Hz}), 7.36(1 \mathrm{H}, \mathrm{d}, J=7.5 \mathrm{~Hz})$, $7.32(2 \mathrm{H}, \mathrm{d}, J=7.0 \mathrm{~Hz}), 7.26(1 \mathrm{H}$, br s$), 5.38(1 \mathrm{H}$, br s $), 5.29(1 \mathrm{H}$, br s), $4.86(1 \mathrm{H}$, br s $), 4.72(1 \mathrm{H}, \mathrm{t}, J=4.5 \mathrm{~Hz}), 1.45(9 \mathrm{H}, \mathrm{s}) ;{ }^{13} \mathrm{C} \mathrm{NMR}$ $\left(125 \mathrm{MHz}, \mathrm{CDCl}_{3}\right) \delta 153.8,136.9,129.0,128.5,126.3,78.7,52.7,28.11$; Chiral HPLC (chiralpak IA column; hexane:iPrOH $90: 10 ; 1.0 \mathrm{~mL} / \mathrm{min}, 210 \mathrm{~nm}$ ) $\mathrm{Rt}_{\text {major }}=12.0 \mathrm{~min}$, $\mathrm{Rt}_{\text {minor }}=13.0 \mathrm{~min}, 91 \%$ ee; Anal. calcd. for $\mathrm{C}_{13} \mathrm{H}_{18} \mathrm{~N}_{2} \mathrm{O}_{4}$ (266.29): C, 58.63; H, 6.81; N, 10.52. Found: C, 58.62; H, 6.81; N, 10.53 .

(2R)-2-(4-Methoxy-phenyl)-1-nitroeth-2-yl-carbamic acid tert-butyl ester (3i)<smiles>COc1ccc(C(C[N+](=O)[O-])NC(=O)OCc2ccccc2)cc1</smiles>

$3 \mathbf{i}$ The title compound was prepared according to the general procedure starting from amido sulfone $1 \mathrm{e}(0.12 \mathrm{~g}, 0.5 \mathrm{mmol})$. Yield $0.12 \mathrm{~g}(82 \%)$; white solid; m.p. $115-116^{\circ} \mathrm{C},[\alpha]_{\mathrm{D}}{ }^{25}=-$ $15.3\left(c=0.5, \mathrm{CHCl}_{3}\right)$; IR $\left(\mathrm{KBr}, \mathrm{cm}^{-1}\right) 3320,2945,1689,1548$; ${ }^{1} \mathrm{H}$ NMR $\left(500 \mathrm{MHz}, \mathrm{CDCl}_{3}\right) \delta 7.23(2 \mathrm{H}, \mathrm{d}, J=8.5 \mathrm{~Hz}), 6.89$ $(2 \mathrm{H}, \mathrm{d}, J=8.6 \mathrm{~Hz}), 5.29(1 \mathrm{H}$, br s $), 4.84(1 \mathrm{H}$, br s $), 5.65(1 \mathrm{H}$, m), $4.47(1 \mathrm{H}, \mathrm{m}), 3.8(3 \mathrm{H}, \mathrm{s}), 1.44(9 \mathrm{H}, \mathrm{s}) ;{ }^{13} \mathrm{C} \mathrm{NMR}\left(125 \mathrm{MHz}, \mathrm{CDCl}_{3}\right) \delta 159.6,154.2$, 127.5, 114.4, 81.5, 78.8, 55.1, 28.1; Chiral HPLC (chiralpak IA column; hexane:iPrOH 90:10; $0.8 \mathrm{~mL} / \mathrm{min} 210 \mathrm{~nm}) \mathrm{Rt}_{\text {major }}=20.0 \mathrm{~min}, \mathrm{Rt}_{\text {minor }}=19.0 \mathrm{~min}, 91 \%$ ee; Anal. calcd. for $\mathrm{C}_{14} \mathrm{H}_{20} \mathrm{~N}_{2} \mathrm{O}_{5}$ (296.32): C, 56.75; H, 6.80; N, 9.45. Found: C, 56.73; H, 6.82; N, 9.45.

\footnotetext{
${ }^{4}$ Nugent, B. M.; Yoder, R. A.; Johnston, J. N. J. Am. Chem. Soc. 2004, 126, 3418-3419.

${ }^{5}$ Yoon, T. P.; Jacobsen, E. N. Angew. Chem. Int. Ed. 2005, 44, 466-468.
} 
(2R)-2-(4-Chloro-phenyl)-1-nitroeth-2-yl-carbamic acid tert-butyl ester (3j)

The title compound was prepared according to the general procedure starting from<smiles>CC(C)(C)OC(=O)NC(C[N+](=O)[O-])c1ccc(Cl)cc1</smiles>

3j amido sulfone $1 \mathbf{j}(0.12 \mathrm{~g}, 0.5 \mathrm{mmol})$. Yield $0.11 \mathrm{~g}(79 \%)$; white solid; m.p. $121-123{ }^{\circ} \mathrm{C}$; $[\alpha]_{\mathrm{D}}{ }^{25}=-25.0\left(c=0.5, \mathrm{CHCl}_{3}\right)$; IR $(\mathrm{KBr}$, $\left.\mathrm{cm}^{-1}\right) 3368,2956,1704,1540 ;{ }^{1} \mathrm{H}$ NMR $\left(500 \mathrm{MHz}, \mathrm{CDCl}_{3}\right) \delta$ $7.40(2 \mathrm{H}, \mathrm{d}, J=1.5 \mathrm{~Hz}), 7.37(1 \mathrm{H}$, br s$), 7.30(2 \mathrm{H}$, br s $), 5.43$ $(1 \mathrm{H}, \mathrm{m}), 4.85(1 \mathrm{H}, \mathrm{m}), 4.74(1 \mathrm{H}, \mathrm{m}), 4.68(1 \mathrm{H}, \mathrm{m}), 1.48(9 \mathrm{H}, \mathrm{s})$; ${ }^{13} \mathrm{C}$ NMR $\left(50 \mathrm{MHz}, \mathrm{CDCl}_{3}\right) \delta 154.8,135.5,134.7,129.4,127.9$, 81.0, 78.8, 52.3, 28.3; Chiral HPLC (chiralpak IA column; hexane:iPrOH 90:10; 1.0 $\mathrm{mL} / \mathrm{min}, 220 \mathrm{~nm}$ ) $\mathrm{Rt}_{\text {major }}=17.0 \mathrm{~min}, \mathrm{Rt}_{\text {minor }}=10.5 \mathrm{~min}, 80 \%$ ee (after crystallization from hexane, $72 \%$ yield; $96 \%$ ee); Anal. calcd. for $\mathrm{C}_{13} \mathrm{H}_{17} \mathrm{ClN}_{2} \mathrm{O}_{4}$ (300.75): C, 59.92; H, 5.70; N, 9.30. Found: C, 59.94; H, 5.68; N, 9.32.

(2R)-1-Nitro-2-(4-trifluoromethyl-phenyl)-eth-2-yl-carbamic acid tert-butyl ester (3k)

The title compound was prepared according to the general procedure starting from<smiles>CC(C)(C)OC(=O)NC(CN=[N+]([O-])[O-])c1ccc(C(F)(F)F)cc1</smiles>
amido sulfone $1 \mathrm{k}(0.14 \mathrm{~g}, 0.5 \mathrm{mmol})$. Yield $0.13 \mathrm{~g}(80 \%)$; white solid (ethyl acetate/hexane); m.p. $121-123{ }^{\circ} \mathrm{C} ;[\alpha]_{\mathrm{D}}{ }^{25}=-$ 10.5 (c 1.0, acetone); ${ }^{1} \mathrm{H}$ NMR $\left(500 \mathrm{MHz}, \mathrm{CDCl}_{3}\right) \delta 7.65(2 \mathrm{H}$, $\mathrm{d}, J=8.2 \mathrm{~Hz}), 7.45(2 \mathrm{H}, \mathrm{d}, J=8.1 \mathrm{~Hz}), 5.5(1 \mathrm{H}, \mathrm{br} \mathrm{s}), 5.45(1 \mathrm{H}$, br s), $4.87(1 \mathrm{H}, \mathrm{m}), 4.73(1 \mathrm{H}, \mathrm{m}), 1.44(9 \mathrm{H}, \mathrm{s}) ;{ }^{13} \mathrm{C}$ NMR $(125$ $\left.\mathrm{MHz}, \mathrm{CDCl}_{3}\right) \delta 154.7,141.0,130.9\left(\mathrm{q}, J^{\mathrm{C}-\mathrm{F}}=130 \mathrm{~Hz}\right), 126.8$,

126.4, 81.1, 78.6, 52.3, 28.2; Chiral HPLC (chiralpak AD column; hexane:iPrOH $90: 10 ; 1.0 \mathrm{~mL} / \mathrm{min}, 210 \mathrm{~nm}$ ) $\mathrm{Rt}_{\text {major }}=17.0 \mathrm{~min}, \mathrm{Rt}_{\text {minor }}=10.5 \mathrm{~min}, 82 \%$ ee (after crystallization from hexane, $75 \%$ yield; $90 \%$ ee); Anal. calcd. for $\mathrm{C}_{14} \mathrm{H}_{17} \mathrm{~F}_{3} \mathrm{~N}_{2} \mathrm{O}_{4}$ (334.11): C, 50.3; H, 5.1; N, 8.4. Found: C, 50.8; H, 5.3; N, 8.0.

(2R)-1-Nitro-2-(3-nitro-phenyl)-eth-2-yl-carbamic acid tert-butyl ester (3l)

The title compound was prepared according to the general procedure starting from<smiles>CC(C)(C)OC(=O)N[C@H](C[N+](=O)[O-])c1cccc([N+](=O)[O-])c1</smiles>
amido sulfone 11 (0.12 g, $0.5 \mathrm{mmol})$. Yield $0.10 \mathrm{~g}(72 \%)$; white solid; m.p. $134-135^{\circ} \mathrm{C}[\alpha]_{\mathrm{D}}=-25.3\left(c=1.0\right.$, acetone) (Lit.: ${ }^{6} \mathrm{mp} \mathrm{140-}$ $142^{\circ} \mathrm{C}, 95 \%$ ee; $[\alpha]_{\mathrm{D}}=-14.8(c=1.0$, acetone $) ;{ }^{1} \mathrm{H}$ NMR $(500 \mathrm{MHz}$, $\left.\mathrm{CDCl}_{3}\right) \delta 8.23-8.21(2 \mathrm{H}, \mathrm{m}), 7.70(1 \mathrm{H}, \mathrm{d}, J=7.8 \mathrm{~Hz}), 7.60(1 \mathrm{H} . \mathrm{dd}$, $\left.J=7.8 \mathrm{~Hz}, J^{\prime}=8.5 \mathrm{~Hz}\right), 5.60(1 \mathrm{H}, \mathrm{d}, J=7.5 \mathrm{~Hz}), 5.48(1 \mathrm{H}, \mathrm{d}, J=5.4$ $\mathrm{Hz}), 4.89(1 \mathrm{H}, \mathrm{m}), 4.80\left(1 \mathrm{H}, \mathrm{dd}, J=13.3 \mathrm{~Hz}, J^{\prime}=4.3 \mathrm{~Hz}\right), 1.46(9 \mathrm{H}$, $\mathrm{s}) ;{ }^{13} \mathrm{C}$ NMR $\left(125 \mathrm{MHz}, \mathrm{CDCl}_{3}\right) \delta 154.2,148.3,139.5,132.5$, 130.8, 123.5, 121.6, 81.5, 78.4, 52.4, 28.4; Chiral HPLC (chiralpak IA column; hexane:iPrOH 90:10;1.0 mL/min, 210nm) $\mathrm{Rt}_{\text {major }}=22.0 \mathrm{~min}, \mathrm{Rt}_{\text {minor }}=14.0 \mathrm{~min}, 83 \%$ ee (after crystallization from hexane, $66 \%$ yield; $90 \%$ ee); Anal. calcd. for $\mathrm{C}_{13} \mathrm{H}_{17} \mathrm{~N}_{3} \mathrm{O}_{6}$ (311.29): C, 50.16; H, 5.50; N, 13.50. Found: C, 50.16; H, 5.51; N, 13.50.

\section{(2R)-(2-Naphthalen-1-yl-1-nitro-eth-2-yl)-carbamic acid tert-butyl ester (3m)}

The title compound was prepared according to the general procedure starting from

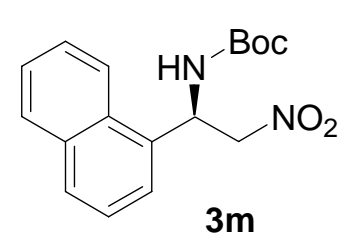
amido sulfone $1 \mathrm{~m}(0.19 \mathrm{~g}, 0.5 \mathrm{mmol})$. Yield $130 \mathrm{mg}(81 \%)$; white solid; m.p. $153-154{ }^{\circ} \mathrm{C}[\alpha]_{\mathrm{D}}{ }^{20}=-9.3\left(c=0.5, \mathrm{CHCl}_{3}\right) ;{ }^{1} \mathrm{H}$ NMR $\left(200 \mathrm{MHz}, \mathrm{CDCl}_{3}\right) \delta 8.15(1 \mathrm{H}, \mathrm{d}, J=8.2 \mathrm{~Hz}), 7.9-7.85(2 \mathrm{H}$, $\mathrm{m}), 7.68-7.53(2 \mathrm{H}, \mathrm{m}), 7.48(2 \mathrm{H}, \mathrm{d}, J=5 \mathrm{~Hz}), 6.32(1 \mathrm{H}, \mathrm{m}), 5.43$ $(1 \mathrm{H}, \mathrm{d}, J=7.8 \mathrm{~Hz}), 4.90(2 \mathrm{H}, \mathrm{d}, J=5.8 \mathrm{~Hz}), 1.46(9 \mathrm{H}, \mathrm{s}) ;{ }^{13} \mathrm{C}$

\footnotetext{
${ }^{6}$ Nugent, B. M.; Yoder, R. A.; Johnston, J. N. J. Am. Chem. Soc. 2004, 126, 3418-3419.
} 
NMR $\left(50 \mathrm{MHz}, \mathrm{CDCl}_{3}\right) \delta 154.8,134.1,132.6,130.3,129.6,129.3,127.3,126.4,125.3$, 123.3, 122.2, 80.8, 78.4, 49.3, 28.3; Chiral HPLC (chiralpak IA column; hexane:iPrOH 90:10; $1.0 \mathrm{~mL} / \mathrm{min}, 220 \mathrm{~nm}$ ) $\mathrm{Rt}_{\text {major }}=13.5 \mathrm{~min}, \mathrm{Rt}_{\text {minor }}=19.5 \mathrm{~min}, 90 \%$ ee (after crystallization from hexane, 74\% yield; $94 \%$ ee); Anal. calcd. for $\mathrm{C}_{17} \mathrm{H}_{20} \mathrm{~N}_{2} \mathrm{O}_{4}$ (316.35): C, 65.54; H, 6.37; N, 8.86. Found: C, 65.49; H, 6.36; N, 8.86.

\section{(2R)-1-Nitro-2-(fur-2-yl)-eth-2-yl-carbamic acid tert-butyl ester (3n)}

The title compound was prepared according to the general procedure starting from

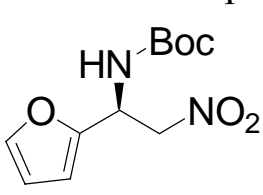

$3 n$ amido sulfone 1n $(0.17 \mathrm{~g}, 0.5 \mathrm{mmol})$. Yield $0.09 \mathrm{~g}(81 \%)$; yellow oil; $[\alpha]_{\mathrm{D}}{ }^{25}=-18.2\left(\right.$ c $\left.1.0, \mathrm{CHCl}_{3}\right) ;{ }^{1} \mathrm{H}$ NMR $\left(500 \mathrm{MHz}, \mathrm{CDCl}_{3}\right) \delta 7.39$ $(1 \mathrm{H}, \mathrm{m}), 6.35-6.27(2 \mathrm{H}, \mathrm{m}), 5.40(1 \mathrm{H}$, br s $), 5.16(1 \mathrm{H}, \mathrm{br} \mathrm{s}), 4.88(1 \mathrm{H}$, m), $4.70(1 \mathrm{H}, \mathrm{m}), 1.45(9 \mathrm{H}, \mathrm{s}) ;{ }^{13} \mathrm{C} \mathrm{NMR}\left(125 \mathrm{MHz}, \mathrm{CDCl}_{3}\right) \delta 154.8$, 149.2, 142.8, 110.2, 107.3, 88.7, 81.0, 47.3, 28.1; Chiral HPLC (chiralpak OD column; hexane:iPrOH 90:10;0.5 mL/min, $210 \mathrm{~nm}$ ) $\mathrm{Rt}_{\text {major }}=20.5 \mathrm{~min}, \mathrm{Rt}_{\text {minor }}=17.5 \mathrm{~min}, 84 \%$ ee; Anal. calcd. for $\mathrm{C}_{11} \mathrm{H}_{16} \mathrm{~N}_{2} \mathrm{O}_{5}$ (256.26): $\mathrm{C}$, 51.56; H, 6.29; N, 10.93. Found: C, 51.43; H, 6.21; N, 10.88 .

\section{(2S,3R)-2-Nitro-5-phenylpent-3-ylcarbamic acid tert-butyl ester (11a)}<smiles>C[C@H](NC(=O)OCc1ccccc1)[C@H](C)[N+](=O)[O-]</smiles>

$11 a$

The title compound was prepared according to the general procedure starting from $\alpha$-amido sulfone $1 \mathrm{a}(0.19 \mathrm{~g}, 0.5 \mathrm{mmol})$. Yield $0.135 \mathrm{~g}(85 \%)$; white solid; $[\alpha]_{\mathrm{D}}{ }^{25}=+15.4$ (c 0.35 , acetone); ${ }^{1} \mathrm{H}$ NMR $\left(200 \mathrm{MHz}, \mathrm{CDCl}_{3}\right) \delta 7.34-7.28(3 \mathrm{H}, \mathrm{m})$, $7.24(2 \mathrm{H}, \mathrm{m}), 5.02(1 \mathrm{H}$, br s $), 4.85-4.64(1 \mathrm{H}, \mathrm{m}), 4.09-3.83(1 \mathrm{H}$, $\mathrm{m}), 2.94-2.49(2 \mathrm{H}, \mathrm{m}), 1.72-1.58(2 \mathrm{H}, \mathrm{m}), 1.52(3 \mathrm{H}, \mathrm{d}, J=6.8$ $\mathrm{Hz}), 1.48(9 \mathrm{H}, \mathrm{s}) ;{ }^{13} \mathrm{C} \mathrm{NMR}\left(125 \mathrm{MHz}, \mathrm{CDCl}_{3}\right) \delta 155.4,140.6,128.6,128.4,126.3$, 85.6, 80.1, 53.3, 32.3, 31.5, 28.3, 15.2; Chiral HPLC (chiralpak IA column; hexane:iPrOH 99:1;0.75 mL/min, 210nm), syn isomer $\mathrm{t}_{\mathrm{r}}$ (major) $=27.5 \mathrm{~min}, \mathrm{t}_{\mathrm{r}}($ minor $)=$ $23.0 \mathrm{~min}$; anti isomer $\mathrm{t}_{\mathrm{r}}=20.5$ and $26.0 \mathrm{~min}$. Isomers ratio as determined by HPLC: syn:anti 90:10; 91\% ee (syn). Anal. calcd. for $\mathrm{C}_{16} \mathrm{H}_{24} \mathrm{~N}_{2} \mathrm{O}_{4}$ (308.37): C, 62.32; H, 7.84; N, 9.08. Found: C, 62.26; H, 7.73; N, 8.98.

\section{(1R,2S)-2-Nitro-1-phenylpropyl carbamic acid tert-butyl ester (11h) ${ }^{6}$}

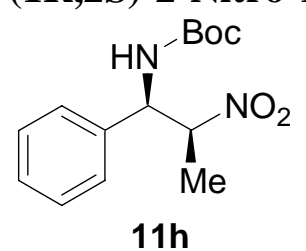

The title compound was prepared according to the general procedure starting from $\alpha$-amido sulfone $\mathbf{1 h}(0.15 \mathrm{~g}, 0.5 \mathrm{mmol})$. Yield $0.123 \mathrm{~g}$ $(88 \%)$; white solid; $[\alpha]_{\mathrm{D}}^{25}=-30.6$ (c 1.0, acetone). (Lit. ${ }^{7}[\alpha]_{\mathrm{D}}{ }^{25}=-$ 26.5 (c 1.0, acetone), 91\% ee); Physical and spectroscopic data consistent with those previously reported. ${ }^{6}{ }^{1} \mathrm{H}$ NMR $(500 \mathrm{MHz}$, $\left.\mathrm{CDCl}_{3}\right) \delta$ 7.33-7.27 (3H, m), 7.24-7.21 (2H, m), $5.33(1 \mathrm{H}$, br s), 5.20 $(1 \mathrm{H}, \mathrm{dd}, \mathrm{J}=8.3,6.0 \mathrm{~Hz}), 4.90(1 \mathrm{H}, \mathrm{br} \mathrm{s}), 1.48(3 \mathrm{H}, \mathrm{d}, \mathrm{J}=6.8 \mathrm{~Hz}), 1.40(9 \mathrm{H}, \mathrm{s}) ;{ }^{13} \mathrm{C}$ NMR (125 MHz, CDCl3) ppm 155.7, 136.5, 129.4, 128.7, 127.3, 86.1, 80.5, 57.5, 28.6, 15.7; Chiral HPLC (chiralpak IA column; hexane:iPrOH 98:2;0.5 mL/min, 210nm), syn isomer $\mathrm{t}_{\mathrm{r}}$ (major) $=47.5 \mathrm{~min}, \mathrm{t}_{\mathrm{r}}($ minor $)=44.5 \mathrm{~min}$; anti isomer $\mathrm{t}_{\mathrm{r}}=56.0$ and $75.5 \mathrm{~min}$. Isomers ratio as determined by HPLC: syn:anti 93:7; 94\% ee (syn). Anal. calcd. for $\mathrm{C}_{14} \mathrm{H}_{20} \mathrm{~N}_{2} \mathrm{O}_{4}$ (280.32): C, 59.99; H, 7.19; N, 9.99. Found: C, 59.49; H, 7.21; N, 10.05.

(1R,2S)-1-(p-Methoxyphenyl)-2-nitropropyl carbamic acid tert-butyl ester (11i) ${ }^{7}$

\footnotetext{
${ }^{7}$ Jacobsen, E.N., Yoon, T.P., Angew. Chem. Int. Ed. 2005,44, 466-468.
} 
<smiles>COc1ccc(C(NC(=O)OCc2ccccc2)C(C)[N+](=O)[O-])cc1</smiles>

The title compound was prepared according to the general procedure starting from $\alpha$-amido sulfone $(0.15 \mathrm{~g}, 0.5 \mathrm{mmol})$. Yield $0.135 \mathrm{~g}(87 \%)$; white solid; $[\alpha]_{\mathrm{D}}{ }^{25}=-22.7$ (c 1.0, acetone) (Lit. ${ }^{7}[\alpha]_{\mathrm{D}}{ }^{25}=-31.2$ (c 1.0, acetone), 96\% ee). Physical and spectroscopic data consistent with those previously reported. ${ }^{7} \mathrm{H}$ NMR $\left(500 \mathrm{MHz}, \mathrm{CDCl}_{3}\right) \delta 7.16(2 \mathrm{H}, \mathrm{d}$ $\mathrm{J}=8.5 \mathrm{~Hz}), 6.87(2 \mathrm{H} \mathrm{d}, \mathrm{J}=8.6 \mathrm{~Hz}), 5.34(1 \mathrm{H}$, br s$), 5.10(1 \mathrm{H}, \mathrm{dd}, \mathrm{J}=6.1,9.1 \mathrm{~Hz}), 4.9$ $\left(1 \mathrm{H}\right.$, br s), $3.78(3 \mathrm{H} \mathrm{s}), 1.54(3 \mathrm{H}, \mathrm{d}, \mathrm{J}=6.8 \mathrm{~Hz}), 1.41(9 \mathrm{H}, \mathrm{s}) ;{ }^{13} \mathrm{C}$ NMR $(125 \mathrm{MHz}$, $\left.\mathrm{CDCl}_{3}\right) \delta 159.8,154.7,127.9,127.5,114.2,86.0,80.5,56.9,55.1,28.0,15.2$; Chiral HPLC (chiralpak AD column; hexane:iPrOH 92:8; $1.0 \mathrm{~mL} / \mathrm{min}, 210 \mathrm{~nm}$ ), syn isomer $\mathrm{t}_{\mathrm{r}}($ major $)=21.0$ min, $\mathrm{t}_{\mathrm{r}}($ minor $)=15.0 \mathrm{~min}$; anti isomer $\mathrm{t}_{\mathrm{r}}=18.0$ and $19.5 \mathrm{~min}$. Isomers ratio as determined by HPLC: syn:anti 95:5; 90\% ee (syn). Anal. calcd. for $\mathrm{C}_{15} \mathrm{H}_{22} \mathrm{~N}_{2} \mathrm{O}_{5}$ (310.35): C, 58.05; H, 7.15; N, 9.03. Found: C, 58.16; H, 7.21; N, 9.11.

\section{(1R,2S)-1-(p-Chlorophenyl)-2-nitropropyl carbamic acid tert-butyl ester (11j) ${ }^{7}$}<smiles>CC(C)(C)OC(=O)N[C@H](c1ccc(Cl)cc1)C([AlH2])[N+](=O)[O-]</smiles>

The title compound was prepared according to the general procedure starting from $\alpha$-amido sulfone $1 \mathbf{j}(0.15 \mathrm{~g}, 0.5 \mathrm{mmol})$. Yield $0.138 \mathrm{~g}(88 \%)$; white solid; $[\alpha]_{\mathrm{D}}{ }^{25}=-31.8$ (c 1.0, acetone) (Lit. $^{7}[\alpha]_{\mathrm{D}}{ }^{25}=-28.3$ (c 1.0, acetone), 95\% ee); Physical and spectroscopic data consistent with those previously reported. ${ }^{7}{ }^{1} \mathrm{H}$ $\operatorname{NMR}(500 \mathrm{MHz}, \mathrm{CDCl} 3) \delta 7.34(2 \mathrm{H}, \mathrm{d}, \mathrm{J}=8.6 \mathrm{~Hz}), 7.17(2 \mathrm{H}, \mathrm{d}$, $\mathrm{J}=7.4 \mathrm{~Hz}), 5.41(1 \mathrm{H}$, br s$), 5.13(1 \mathrm{H}, \mathrm{dd}, \mathrm{J}=8.8,5.7 \mathrm{~Hz}), 4.91(1 \mathrm{H}, \mathrm{br} \mathrm{s}), 1.52(3 \mathrm{H}, \mathrm{d}, \mathrm{J}$ $=7.0 \mathrm{~Hz}), 1.43(9 \mathrm{H}, \mathrm{s}) ;{ }^{13} \mathrm{C} \mathrm{NMR}\left(125 \mathrm{MHz}, \mathrm{CDCl}_{3}\right) \mathrm{ppm} 155.2,135.0,134.6,129.2$, 128.3, 85.6, 81.2, 57.2, 28.3, 15.5; Chiral HPLC (chiralpak AD column; hexane:iPrOH $92: 8 ; 1.0 \mathrm{~mL} / \mathrm{min}, 210 \mathrm{~nm})$, syn isomer $\mathrm{t}_{\mathrm{r}}$ (major) $=20.5 \mathrm{~min}, \mathrm{t}_{\mathrm{r}}($ minor $)=18.0 \mathrm{~min}$; anti isomer $\mathrm{t}_{\mathrm{r}}=22.5$ and $27.0 \mathrm{~min}$. Isomers ratio as determined by HPLC: syn:anti 75:25; 98\% ee (syn). Anal. calcd. for $\mathrm{C}_{14} \mathrm{H}_{19} \mathrm{~N}_{2} \mathrm{O}_{4}$ (314.76): C, 53.42; $\mathrm{H}, 6.08 ; \mathrm{N}, 8.90$. Found: C, 53.28; H, 6.13; N, 8.87.

\section{C) Large scale reaction (10 $\mathrm{mmol})$ :}

(R)-1-Nitropent-2-yl carbamic acid tert-butyl ester (3c).

To the mixture of $\alpha$-amido sulfone 1c $(3.2 \mathrm{~g}, 10 \mathrm{mmol})$ and $\mathrm{N}$-benzyl
quininium chloride $5(540 \mathrm{mg}, 1.2 \mathrm{mmol})$ in toluene $(30 \mathrm{~mL})$ were
added successively $\mathrm{CH}_{3} \mathrm{NO}_{2}(2.6 \mathrm{~mL}, 50 \mathrm{mmol})$ and, after cooling to stirred at the same temperature for 40 hours and then it was quenched with $\mathrm{HCl}(30 \mathrm{~mL}$, $0.1 \mathrm{~N})$ and extracted with $\mathrm{CH}_{2} \mathrm{Cl}_{2}(3 \times 20 \mathrm{~mL})$. The organic layer was washed with $\mathrm{HCl}$ $(1 \times 30 \mathrm{~mL}, 0.1 \mathrm{~N})$, dried over $\mathrm{MgSO}_{4}$, and concentrated under reduced pressure. The crude product was purified by flash column chromatography using ethyl acetate/hexane mixtures as the eluant. Yield $2.1 \mathrm{~g}(90 \%) ; 97 \%$ ee. 


\section{D) Assignment of the absolute configuration of adducts}

The absolute configuration of aromatic adducts $\mathbf{3 h}, \mathbf{l}$ was deduced from comparison of the optical rotation values with published data. For details, see characterization data of each compound above.

The assignment of the absolute, and syn/anti, configuration of aromatic adducts $\mathbf{1 1 h}-\mathbf{j}$ was made by comparison of HPLC retention times with published values (see the chromatograms below). The absolute, and syn/anti, configuration of (aliphatic) adduct 11a was made by assuming a uniform reaction mechanism.

\section{Absolute configuration of 3c:}

Method A: Through chemical correlation to 1,2-diamine dihydrochloride 12: ${ }^{8}$<smiles>CCCC(N)CN</smiles>

12

To a solution of 3c $(0.232 \mathrm{~g}, 1 \mathrm{mmol})$ in methanol $(1.5 \mathrm{~mL})$ was added $12 \mathrm{~N} \mathrm{HCl}(0.3 \mathrm{~mL})$ and activated $\mathrm{Pd} / \mathrm{C}(10 \%, 0.023$ g) and, after removing of air with vaccum, the mixture was kept under hydrogen atmosphere at room temperature until the starting material disappeared $(20 \mathrm{~h})$. The resulting suspension was filtered through a short pad of Celite, which was then washed with methanol $(5 \mathrm{~mL})$ and the solution concentrated under reduced pressure. A white solid was obtained: yield $157 \mathrm{mg}(90 \%)$; $[\alpha]_{\mathrm{D}}{ }^{25}=+10.8(c 1.0, \mathrm{MeOH}),\left[\mathrm{Lit}^{8}[\alpha]_{\mathrm{D}}{ }^{25}=+10.5\right.$ (c 1.0, MeOH)]. ${ }^{1} \mathrm{H}$ NMR $(200$ $\left.\mathrm{MHz}, \mathrm{CD}_{3} \mathrm{OD}\right) \delta 4.89(6 \mathrm{H}$, br s), 3.58-3.46 (1H, m), 3.32-3.23 $(2 \mathrm{H}, \mathrm{m}), 1.8-1.38(4 \mathrm{H}$, $\mathrm{m}), 0.98(3 \mathrm{H}, \mathrm{t}, J=7.5 \mathrm{~Hz}) ;{ }^{13} \mathrm{C}$ NMR $\left(125 \mathrm{MHz}, \mathrm{CD}_{3} \mathrm{OD}\right) \delta 50.7,42.2,33.6,19.2,13.8$.

Method B: Through chemical correlation to $N, N$-di-Boc-derivative 13: ${ }^{9}$

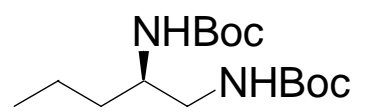

13

To a solution of adduct 3c $(0.93 \mathrm{~g}, 4 \mathrm{mmol})$ in $\mathrm{MeOH}(4 \mathrm{~mL})$ was added $\mathrm{Pd} / \mathrm{C}(10 \%, 92.9 \mathrm{mg})$ and, after removing of air with vaccum, the mixture was kept under hydrogen atmosphere at room temperature overnight. The resulting suspension was filtered through a short pad of Celite and the solution was concentrated in the rotary evaporator. From the resulting crude residue $(808 \mathrm{mg}, 99 \%)$ an aliquot of $0.20 \mathrm{~g}(1$ mmol) was dissolved in $\mathrm{CH}_{2} \mathrm{Cl}_{2}(2 \mathrm{~mL})$ and (Boc) $)_{2} \mathrm{O}(0.33 \mathrm{~g}, 1.5 \mathrm{mmol})$ was added at room temperature and the solution stirred at the same temperature for 2 additional hours. The crude product obtained after evaporation of the solvent was purified by column chromatography on silica gel affording $287 \mathrm{mg}(95 \%)$ of the di-Boc protected 1,2-diamine. $[\alpha]_{\mathrm{D}}{ }^{25}=+30.8\left(c 1.0, \mathrm{CHCl}_{3}\right)$, [Lit. (S enantiomer) $:^{9}[\alpha]_{\mathrm{D}}{ }^{25}=-27.8(c 1.06$, $\left.\left.\mathrm{CHCl}_{3}\right)\right] .{ }^{1} \mathrm{H}$ NMR $\left(200 \mathrm{MHz}, \mathrm{C}_{6} \mathrm{D}_{6}\right) \delta 5.02-4.98(1 \mathrm{H}, \mathrm{m}), 4.88-4.71(1 \mathrm{H}, \mathrm{m}), 3.61-3.46$ $(1 \mathrm{H}, \mathrm{m}), 3.24-3.10(2 \mathrm{H}, \mathrm{m}), 1.44(18 \mathrm{H}, \mathrm{s}), 1.51-1.40(2 \mathrm{H}, \mathrm{m}), 0.95(3 \mathrm{H}, \mathrm{t}, J=7.4 \mathrm{~Hz}) ;{ }^{13} \mathrm{C}$ NMR $\left(125 \mathrm{MHz}, \mathrm{C}_{6} \mathrm{D}_{6}\right) \delta 156.7,156.2,79.2,52.8,44.6,29.1,28.4,26.2,10.8$.

\footnotetext{
${ }^{8}$ Lucet, D.; Sabell, S.; Kostelitz, O.; Le Gall, T.; Mioskowski, C. Eur. J. Org. Chem. 1999, 2583-2591.

${ }^{9}$ Enders, D.; Wiedemann, J. Synthesis 1996, 1443-1450.
} 
E) ${ }^{1} \mathrm{H}$ and ${ }^{13} \mathrm{C}$ NMR data of selected compounds
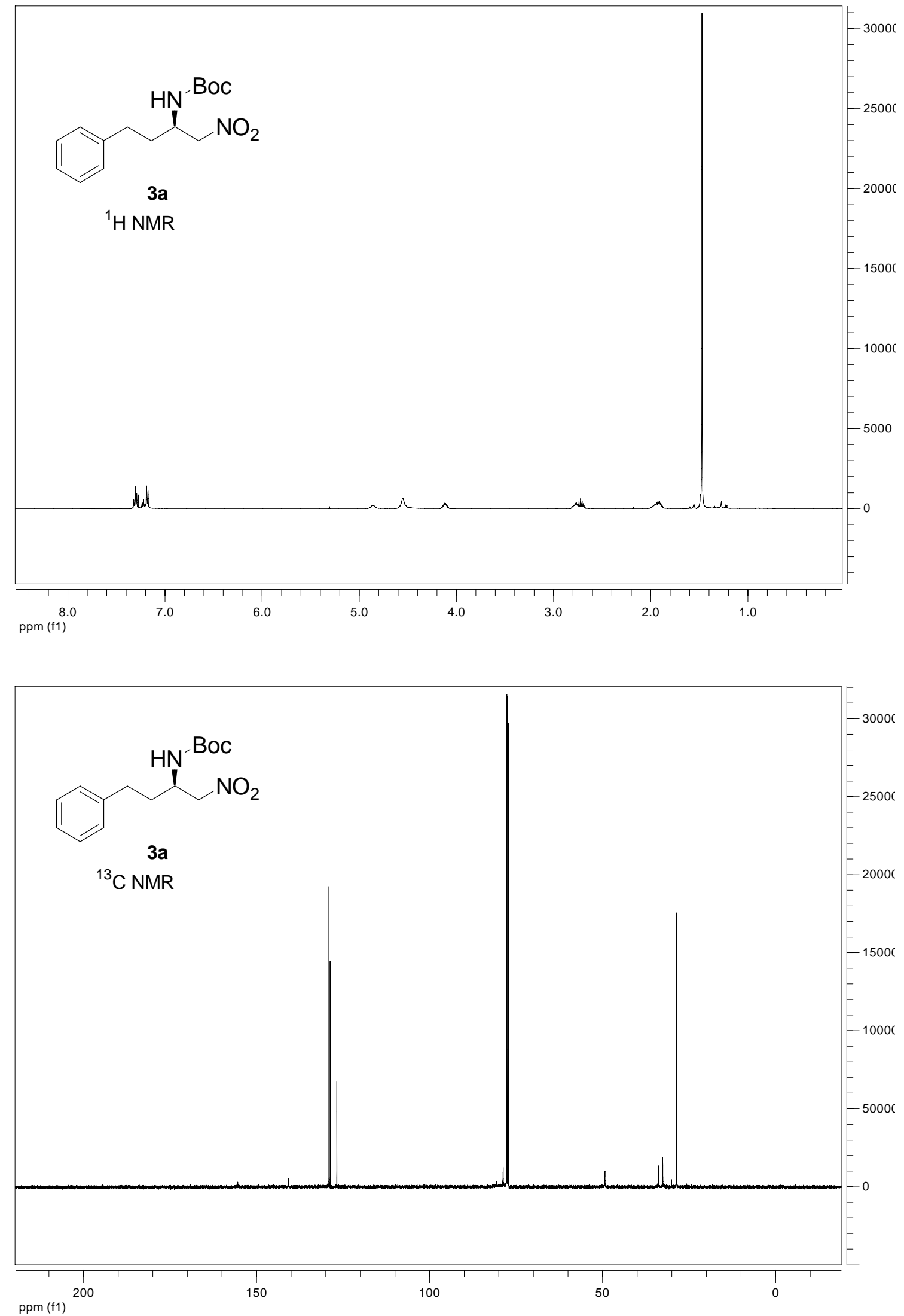

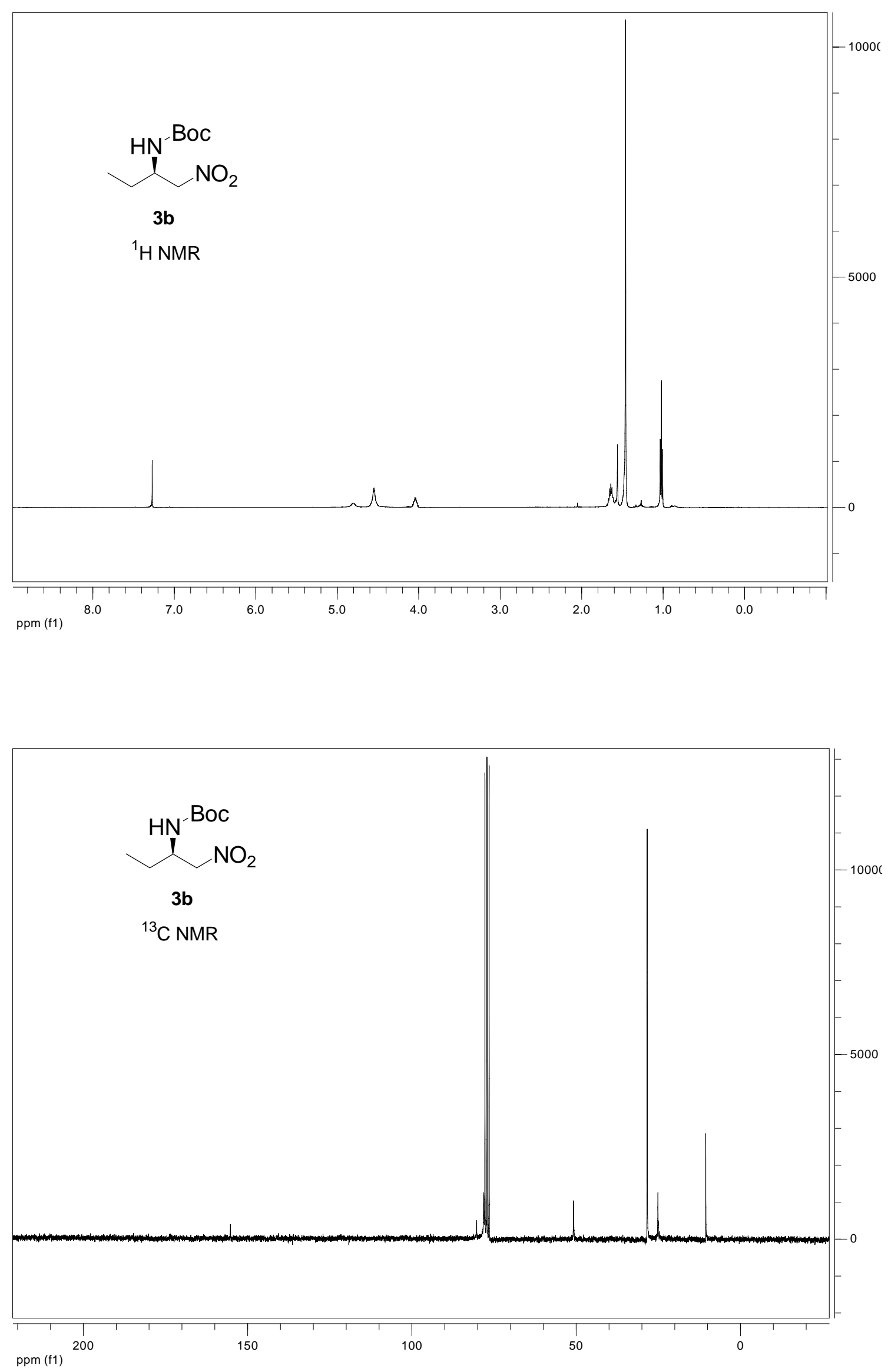

S10 


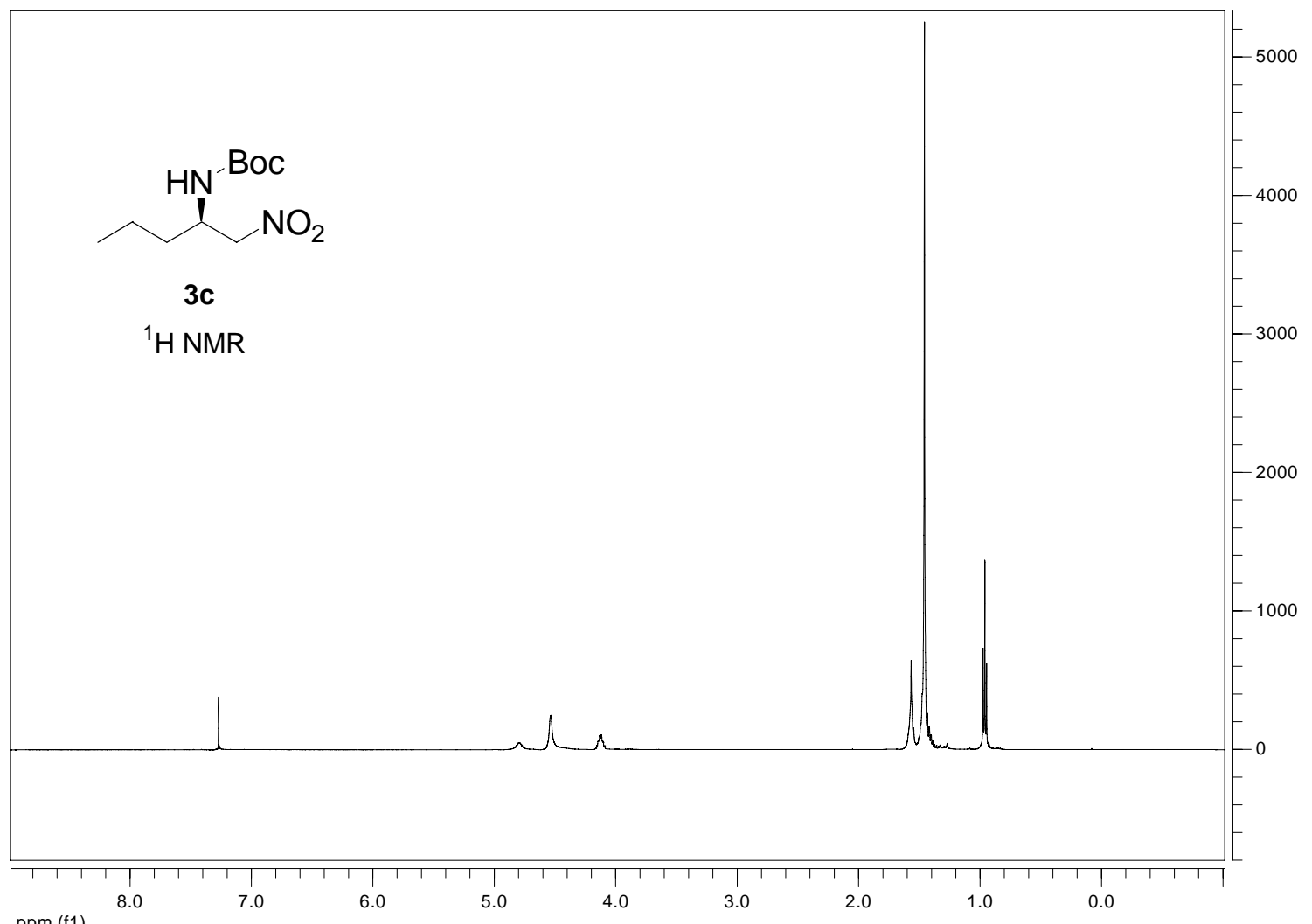

ppm (f1)

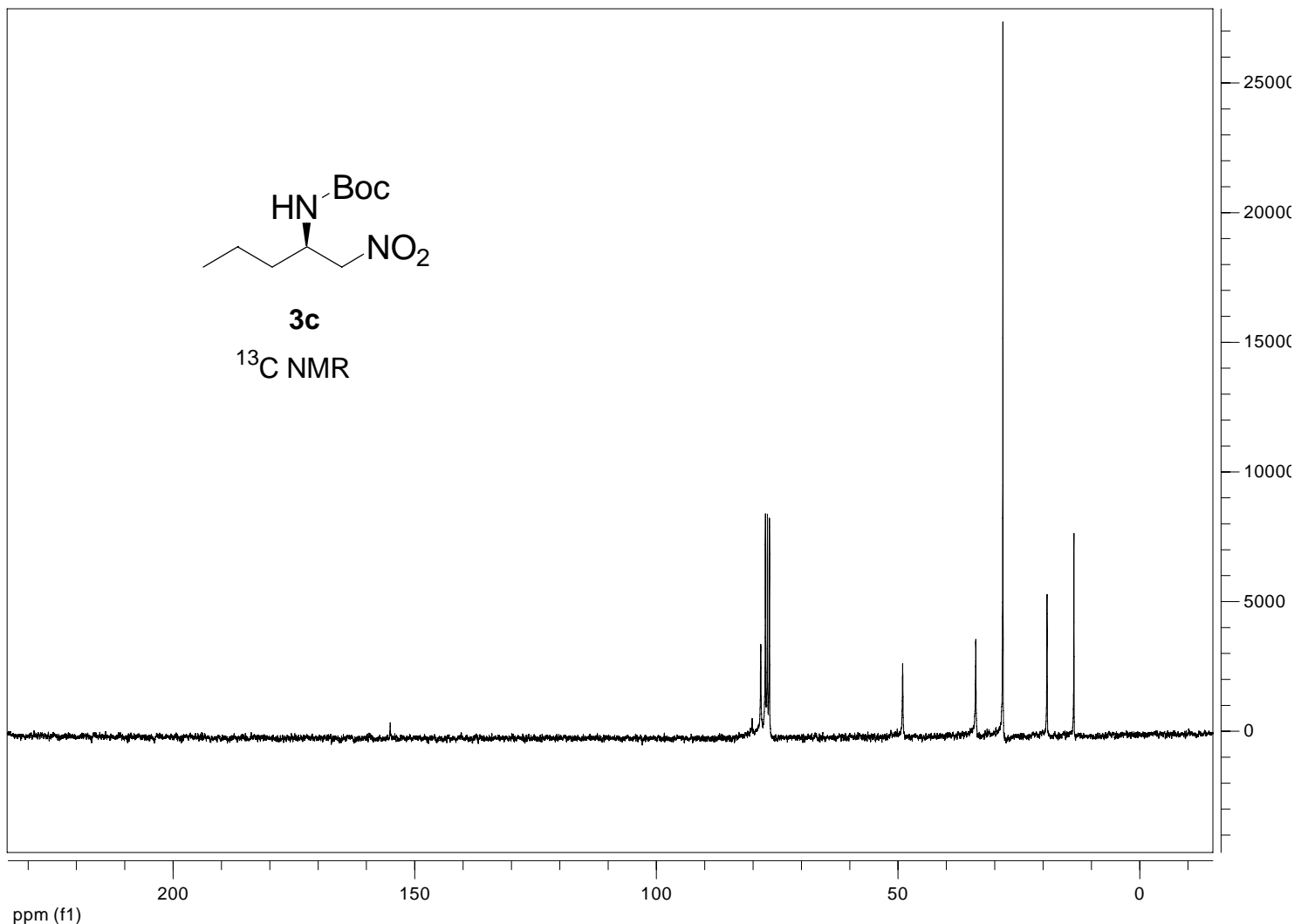




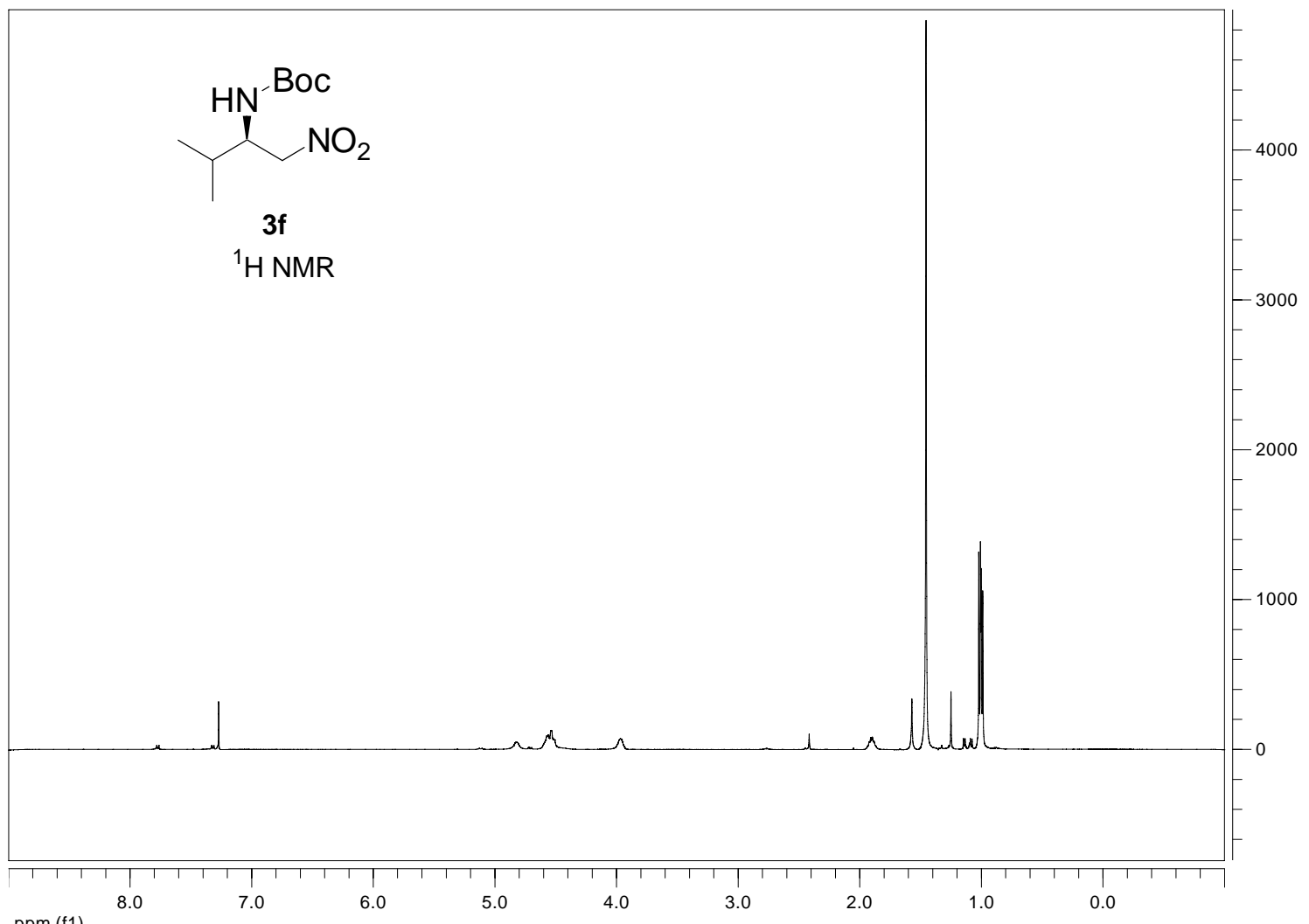

ppm (f1)

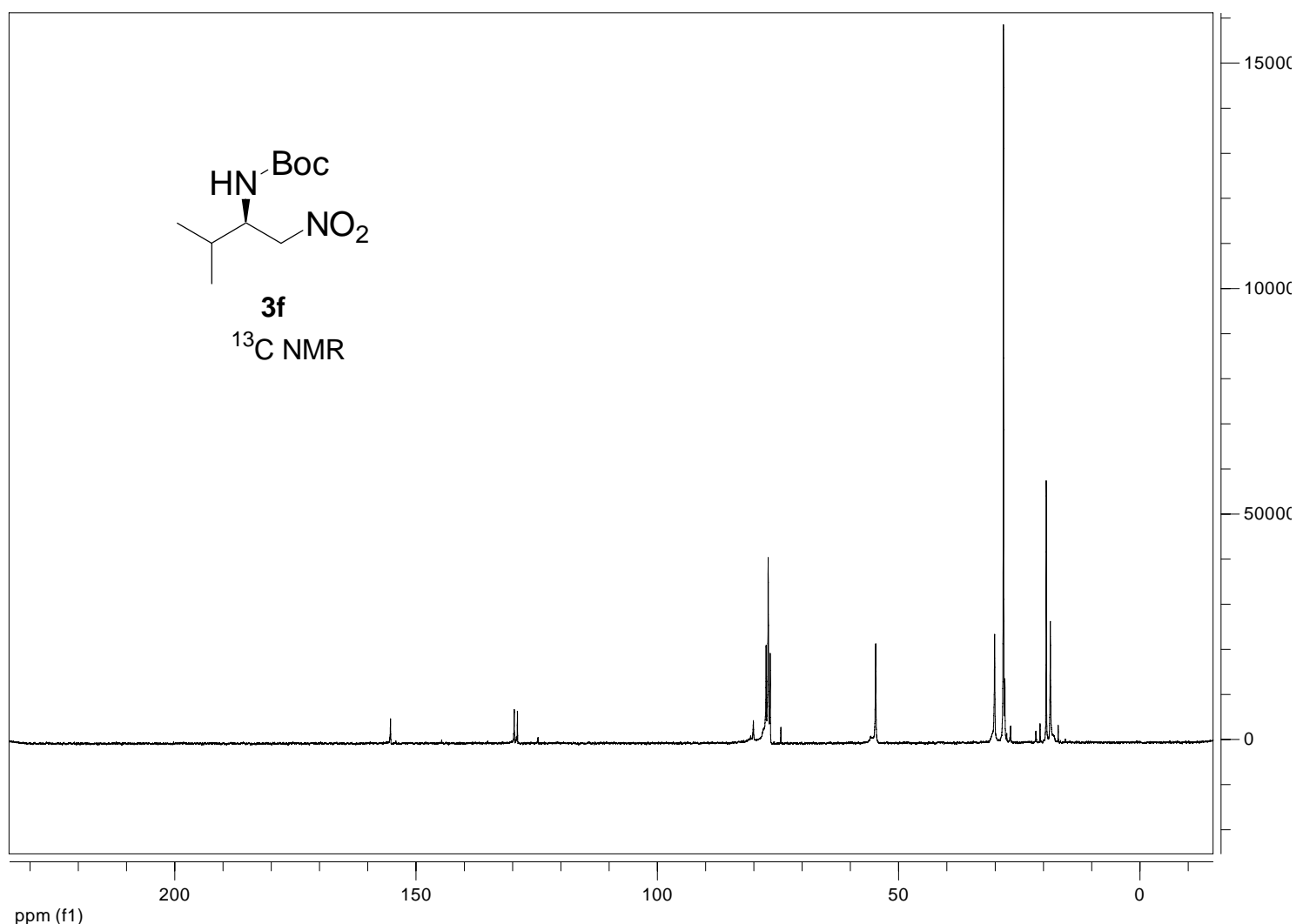



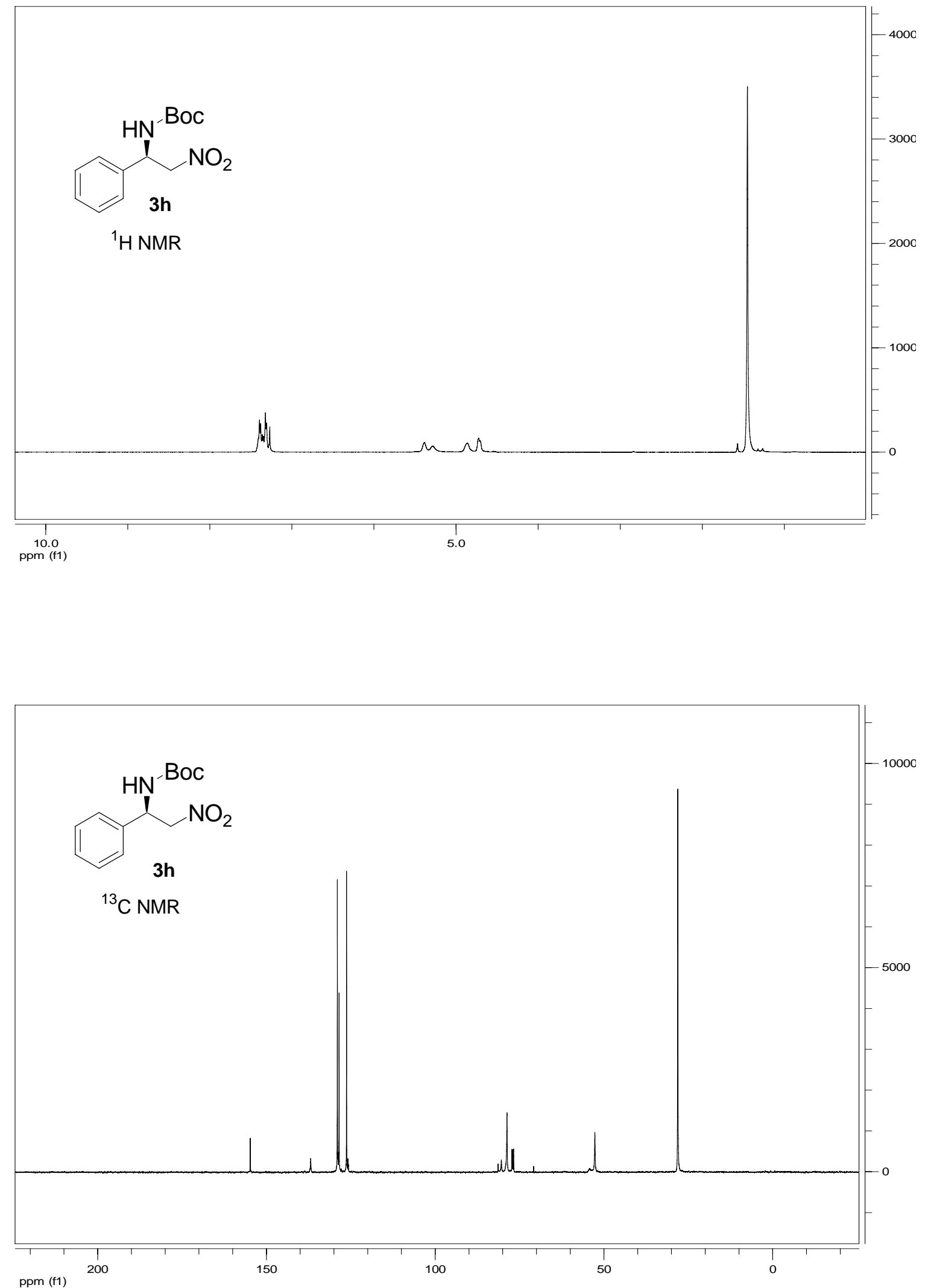

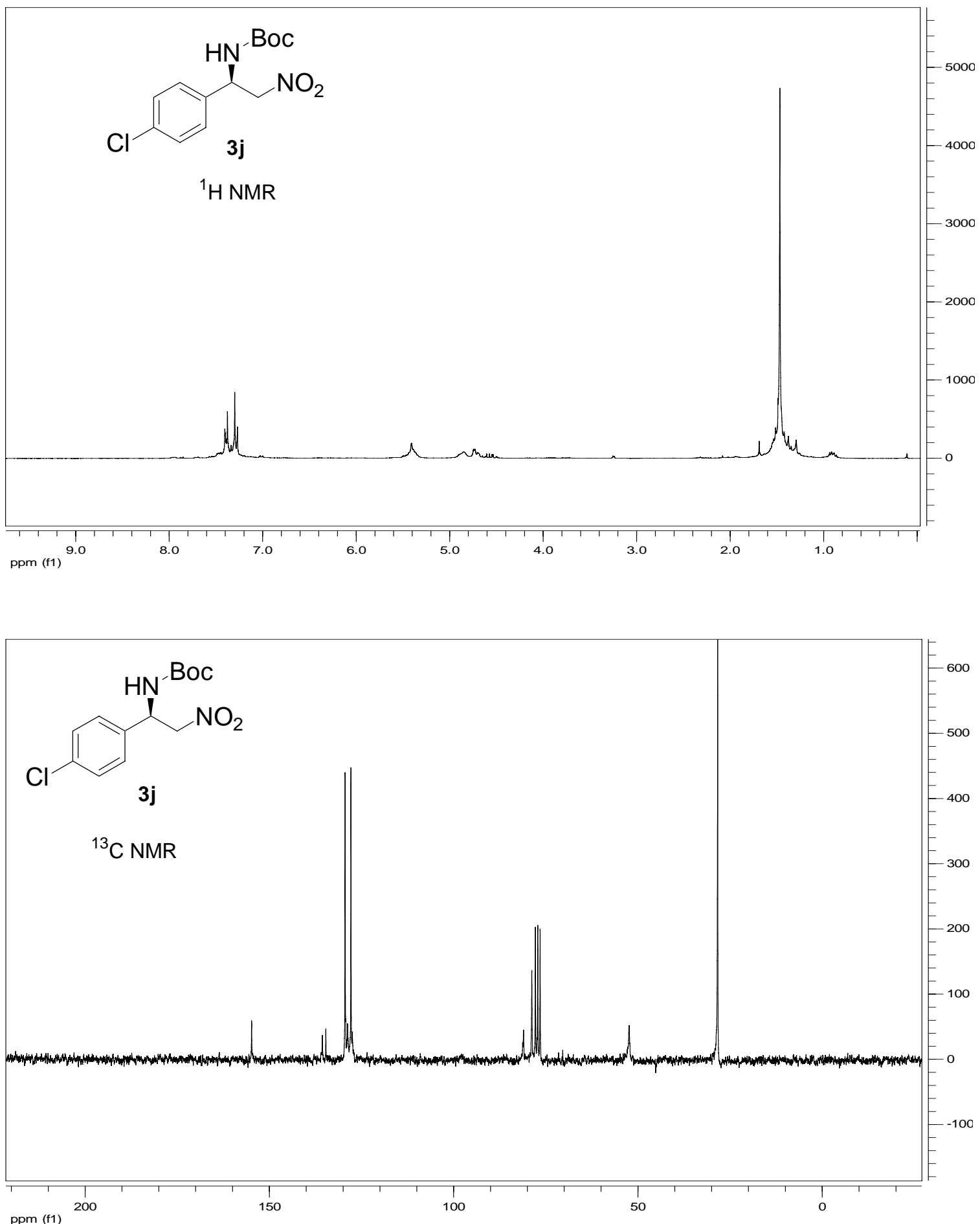

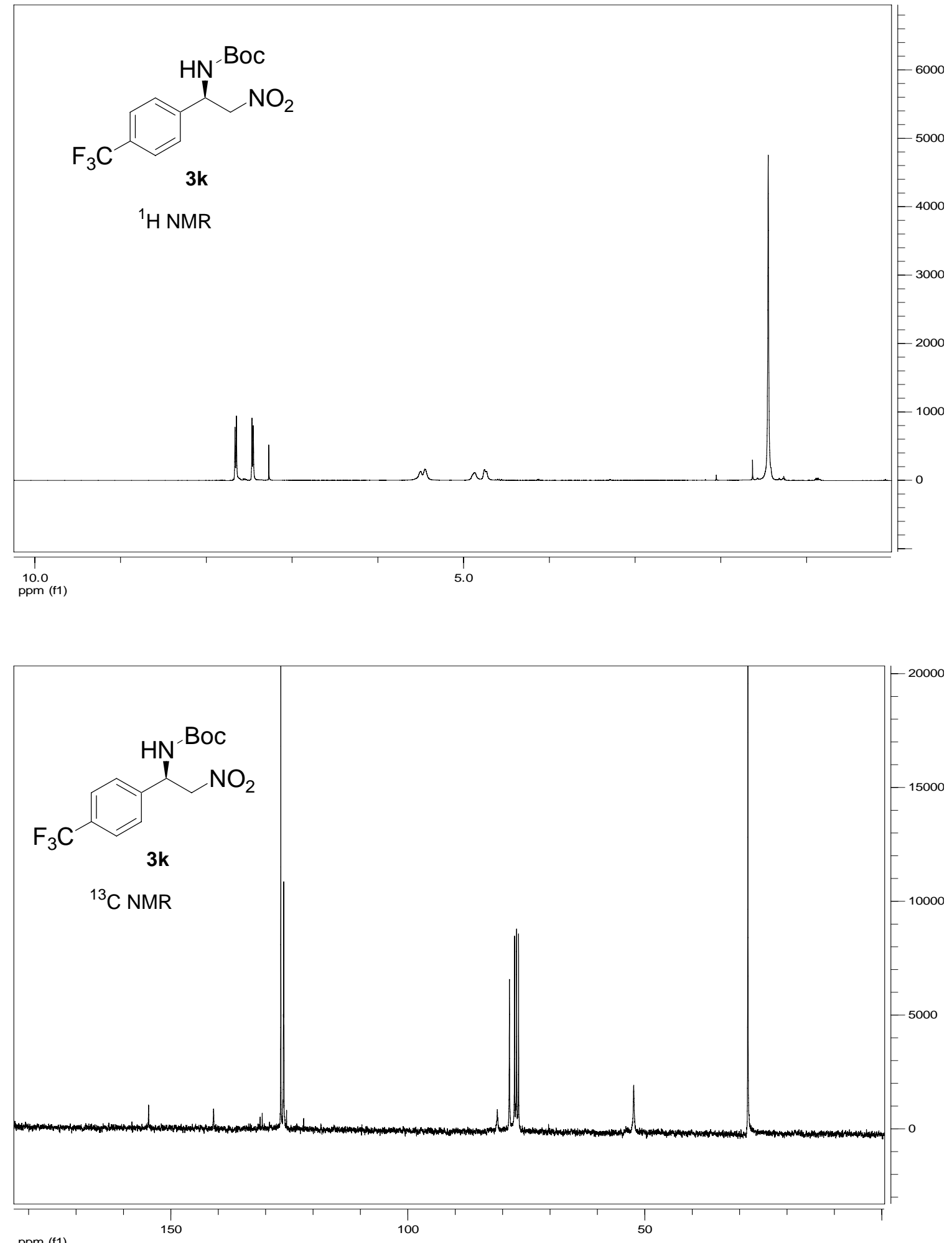

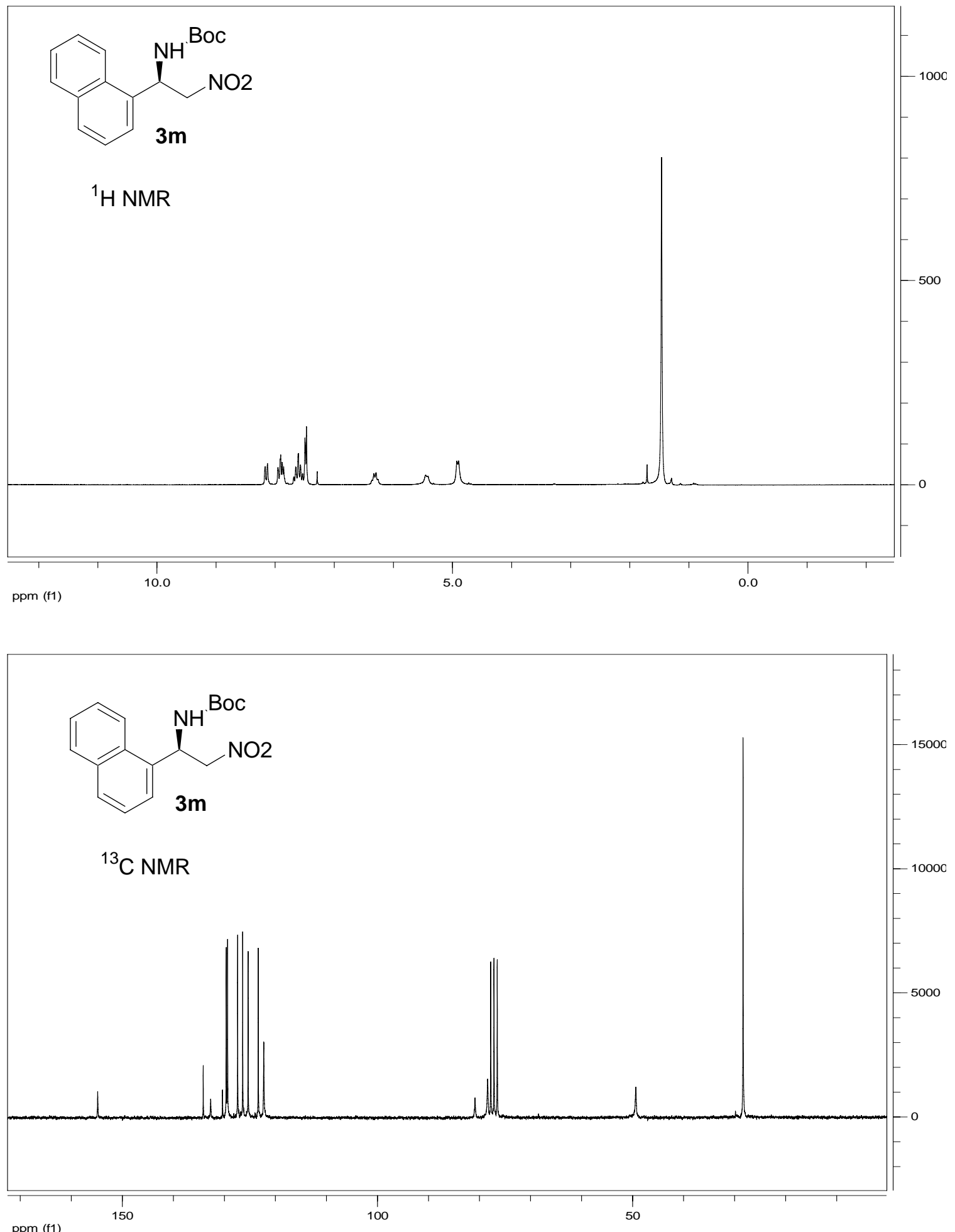


\section{F) HPLC chromatograms of selected products}

Chiralpak AD, 98:2 hexane:iPrOH, $1 \mathrm{~mL} / \mathrm{min}, \lambda=210 \mathrm{~nm}$
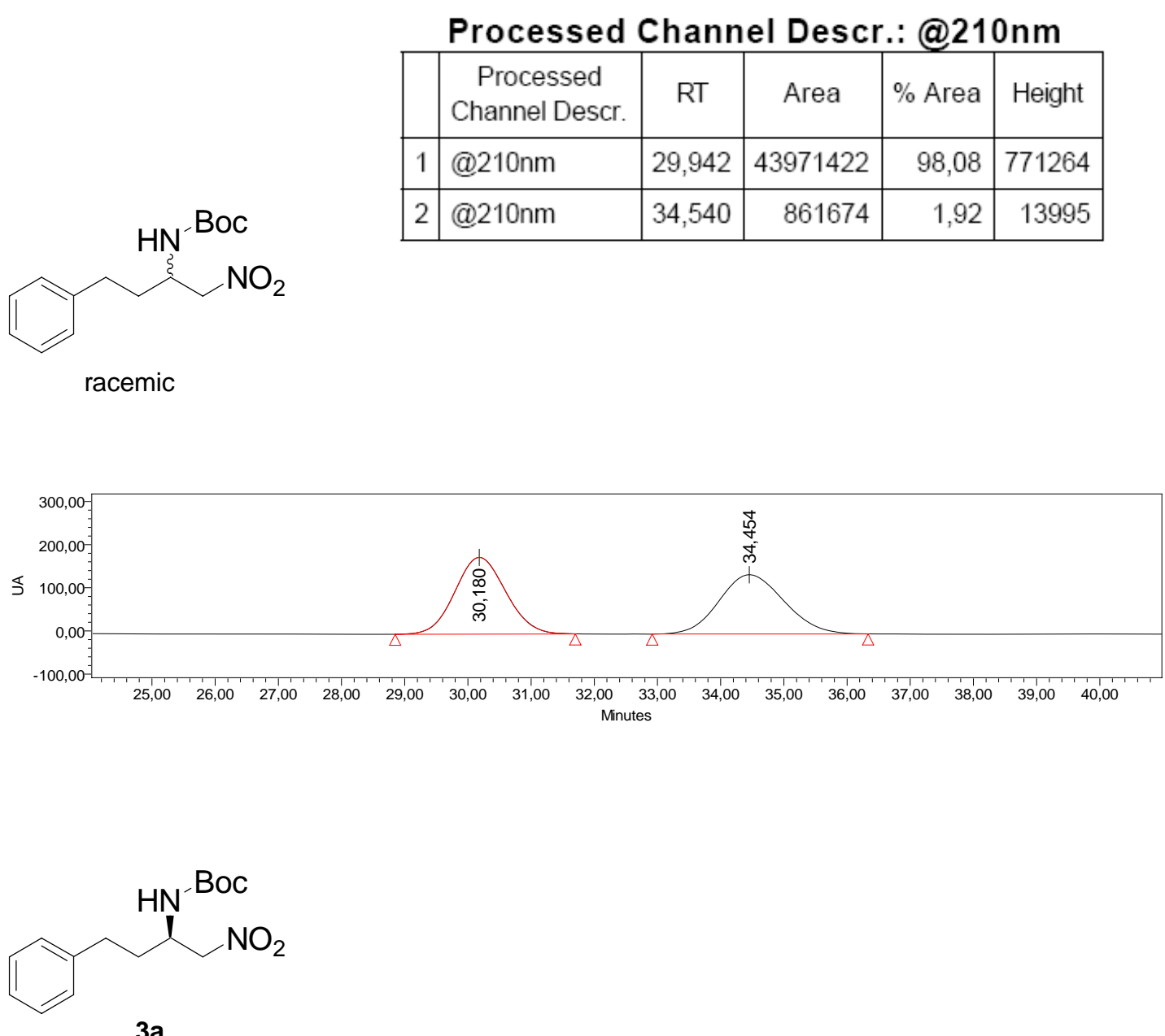

$3 a$

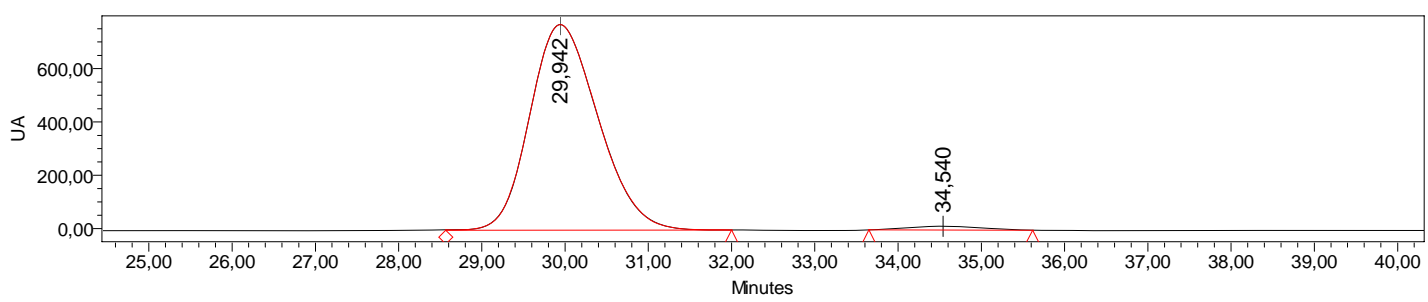


Chiralpak AD, 95:5 hexane:iPrOH, $1 \mathrm{~mL} / \mathrm{min}, \lambda=254 \mathrm{~nm}$

Processed Channel Descr.: @254nm

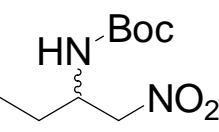

\begin{tabular}{|l|l|c|r|r|r|}
\hline & $\begin{array}{c}\text { Processed } \\
\text { Channel Descr. }\end{array}$ & RT & Area & $\%$ Area & Height \\
\hline 1 & $@ 254 \mathrm{~nm}$ & 10,177 & 524884 & 97,95 & 29311 \\
\hline 2 & $@ 254 \mathrm{~nm}$ & 12,453 & 10965 & 2,05 & 476 \\
\hline
\end{tabular}

racemic

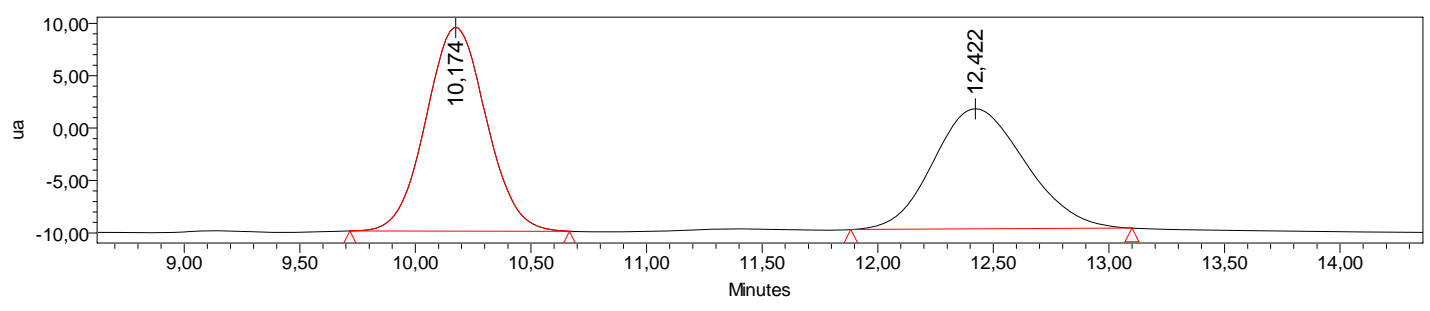

$\mathrm{NO}_{2}$

$3 b$

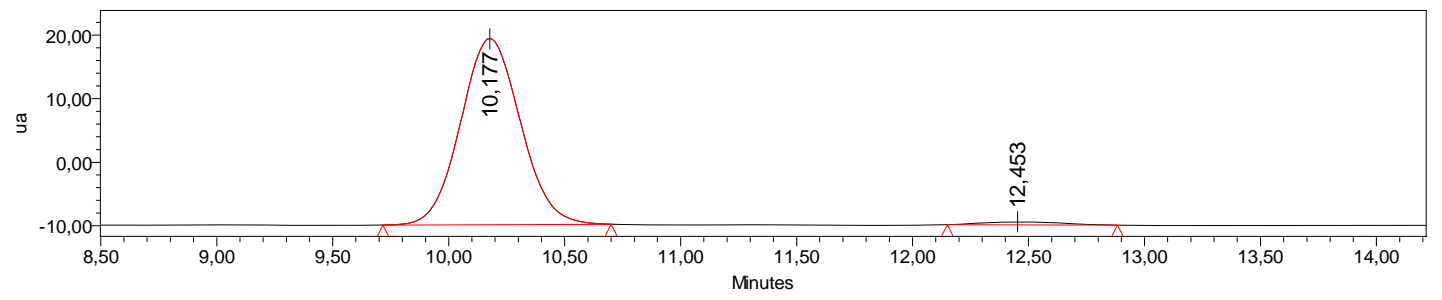


Chiralpak AD, 90:10 hexane:iPrOH, $0.8 \mathrm{~mL} / \mathrm{min}, \lambda=210 \mathrm{~nm}$

Processed Channel Descr.: @210nm
\begin{tabular}{r|l|c|c|r|r|}
\hline $\begin{array}{c}\text { Processed } \\
\text { Channel Descr. }\end{array}$ & RT & \multicolumn{1}{c|}{ Area } & $\%$ Area & Height \\
\hline 1 & $@ 210 \mathrm{~nm}$ & 8,736 & 24072661 & 98,76 & 1747387 \\
\hline 2 & $@ 210 \mathrm{~nm}$ & 9,928 & 303420 & 1,24 & 19037 \\
\hline
\end{tabular}<smiles>CCC[C@H](CNC(=O)OCc1ccccc1)NC(=O)OCc1ccccc1</smiles>

racemic

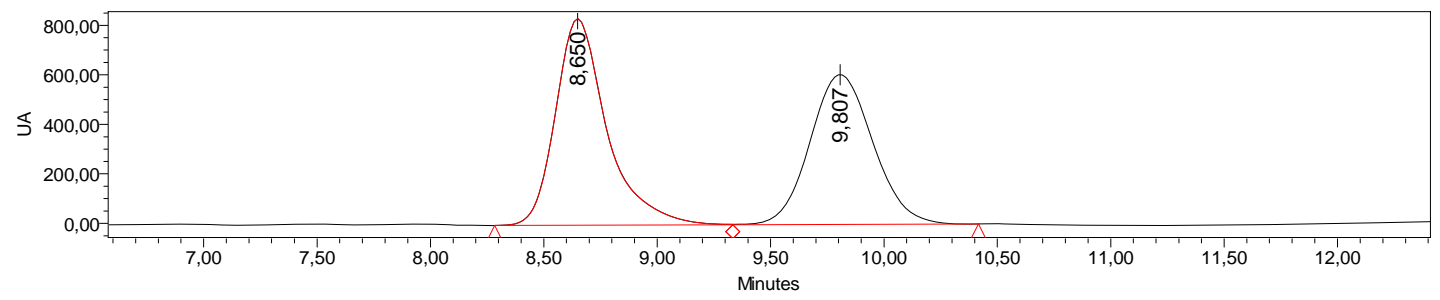

$\mathrm{NO}_{2}$

(10 mmol scale reaction)

$3 c$

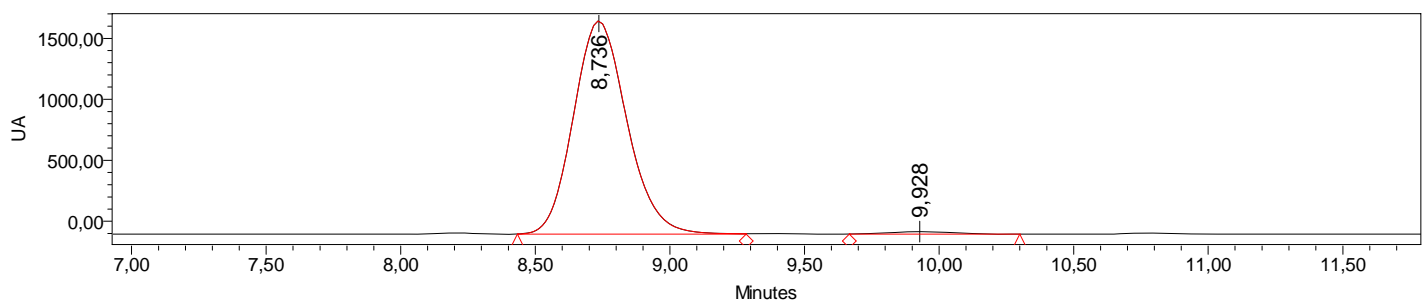


Chiralpak AD, 95:5 hexane:iPrOH, $1 \mathrm{~mL} / \mathrm{min}, \lambda=210 \mathrm{~nm}$
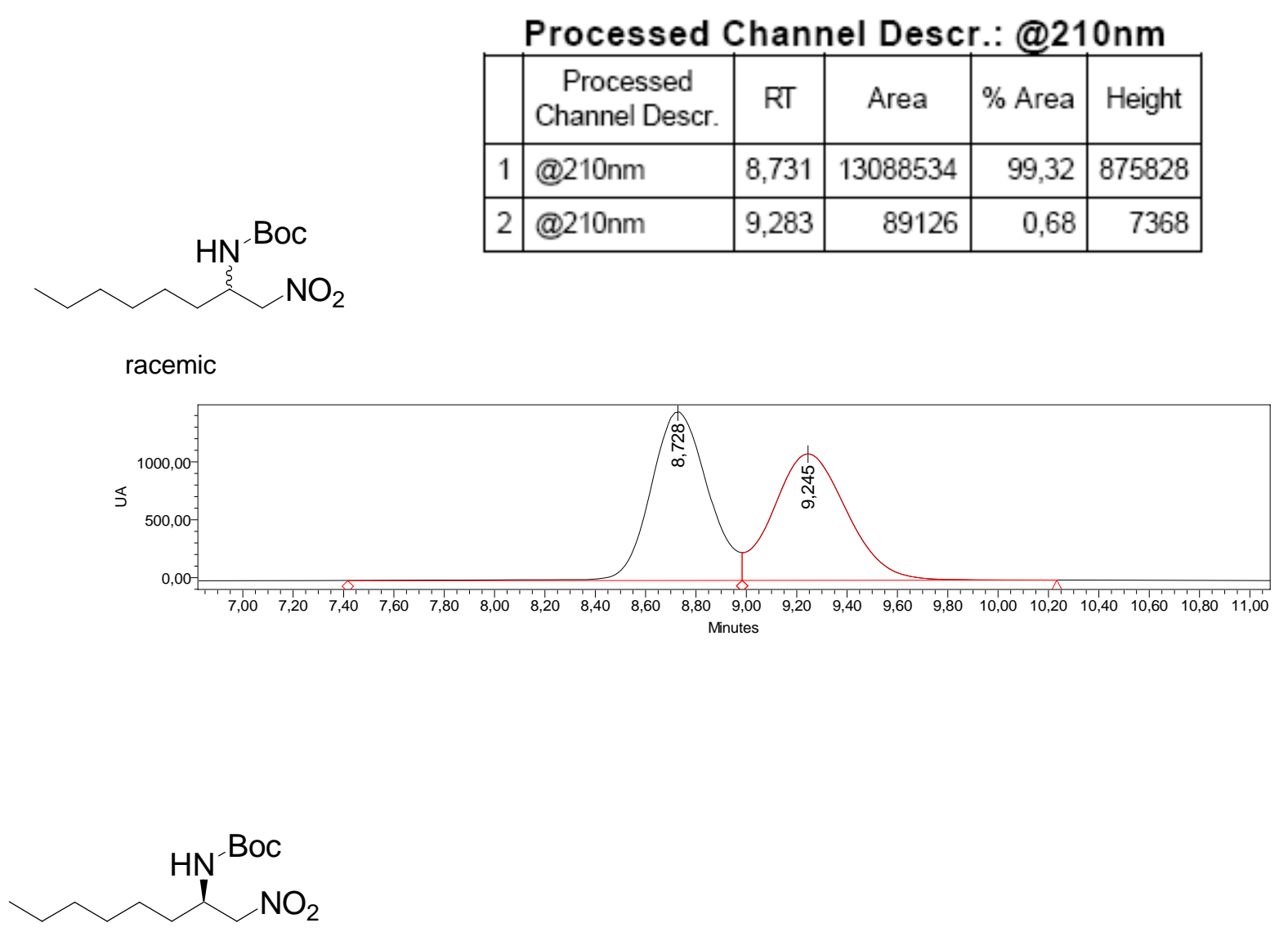

3d

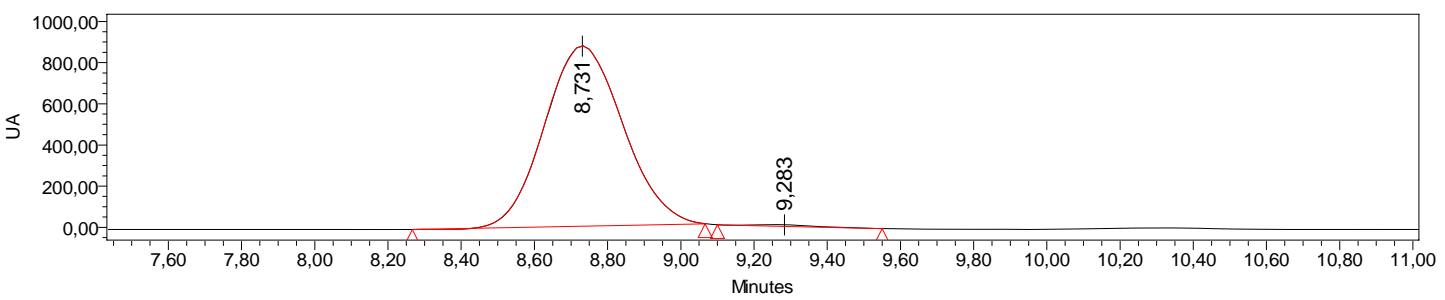


Chiralpak AD, 90:10 hexane:iPrOH, $0.8 \mathrm{~mL} / \mathrm{min}, \lambda=210 \mathrm{~nm}$

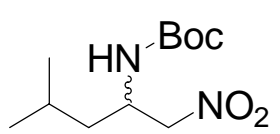

Processed Channel Descr.: @210nm
\begin{tabular}{|l|l|c|r|r|r|}
\hline $\begin{array}{c}\text { Processed } \\
\text { Channel Descr. }\end{array}$ & RT & Area & $\%$ Area & Height \\
\hline 1 & $@ 210 \mathrm{~nm}$ & 8,521 & 847370 & 98,79 & 62876 \\
\hline 2 & $@ 210 \mathrm{~nm}$ & 9,867 & 10417 & 1,21 & 752 \\
\hline
\end{tabular}

racemic
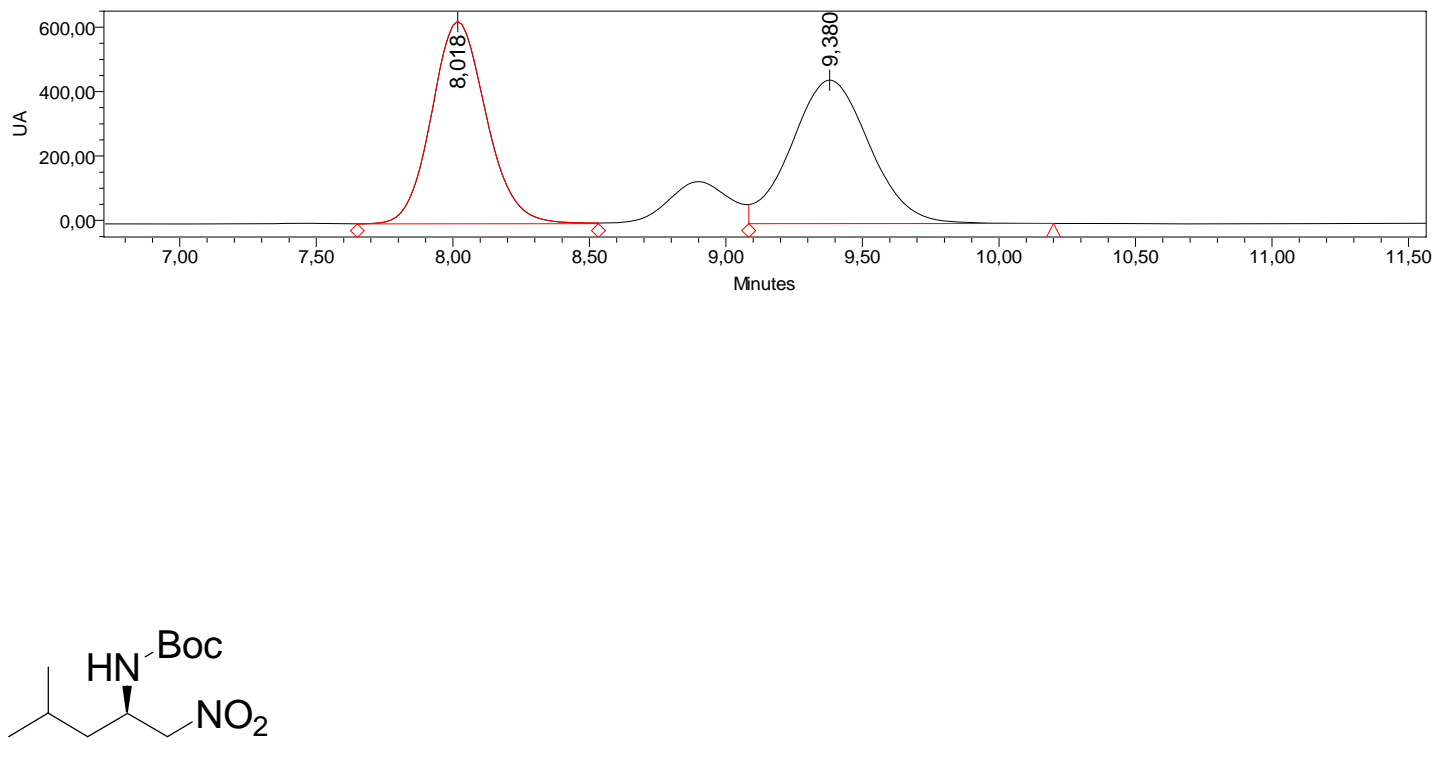

$3 e$

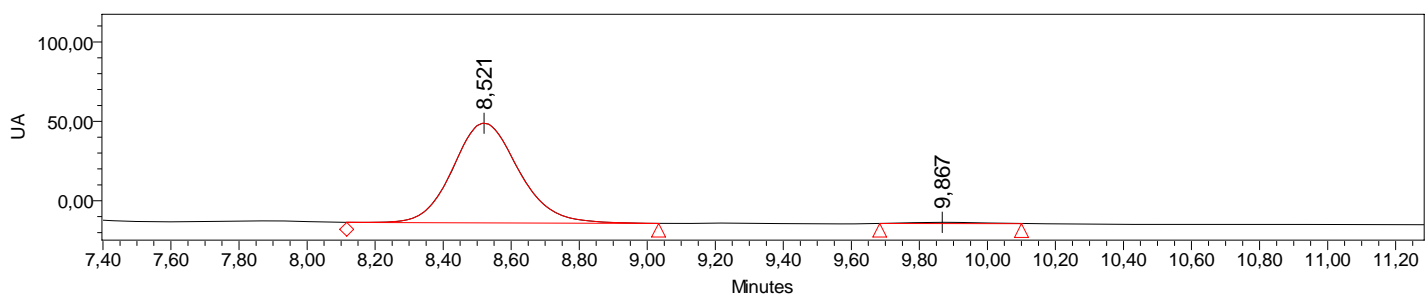


Chiralpak AD, 90:10 hexane:iPrOH, $0.8 \mathrm{~mL} / \mathrm{min}, \lambda=210 \mathrm{~nm}$
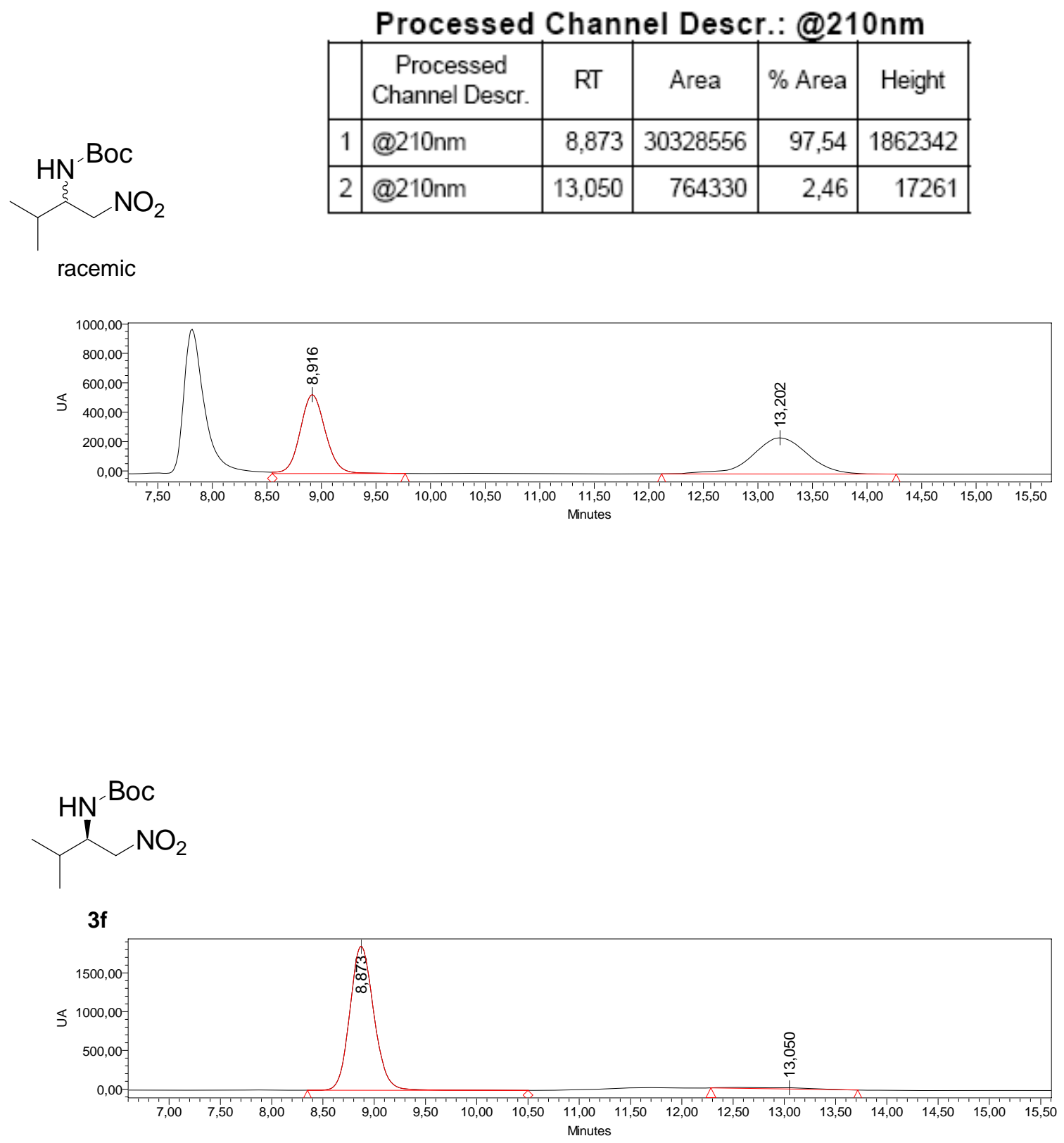
Chiralpak AD, 90:10 hexane:iPrOH, $1 \mathrm{~mL} / \mathrm{min}, \lambda=210 \mathrm{~nm}$

Processed Channel Descr.: @210nm

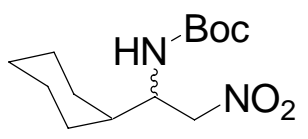

\begin{tabular}{|r|l|c|r|r|r|}
\hline & $\begin{array}{c}\text { Processed } \\
\text { Channel Descr. }\end{array}$ & RT & \multicolumn{1}{|c|}{ Area } & $\%$ Area & Height \\
\hline 1 & $@ 210 \mathrm{~nm}$ & 8,196 & 4375868 & 98,97 & 316874 \\
\hline 2 & $@ 210 \mathrm{~nm}$ & 9,590 & 45543 & 1,03 & 2313 \\
\hline
\end{tabular}

racemic
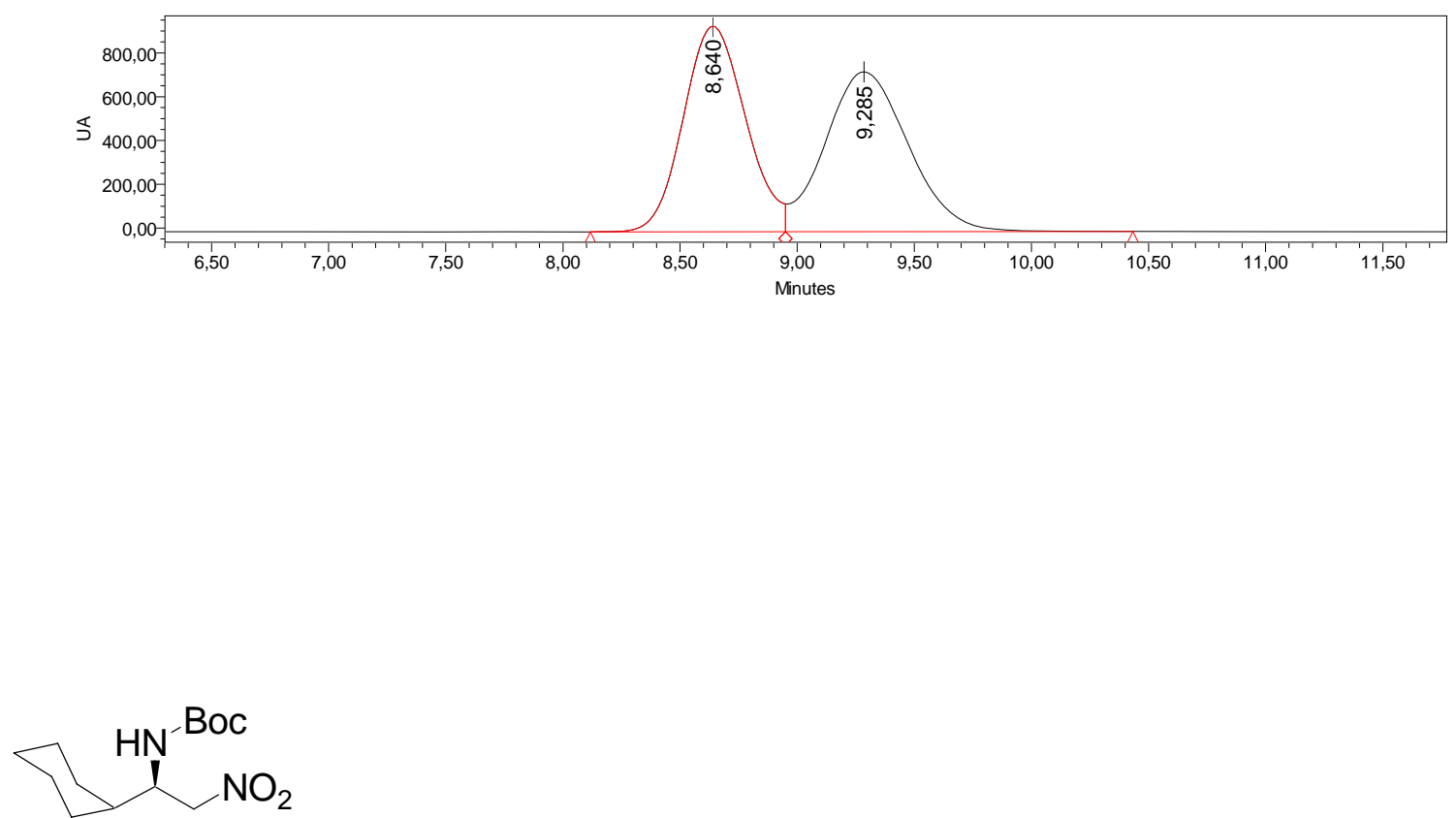

$3 g$

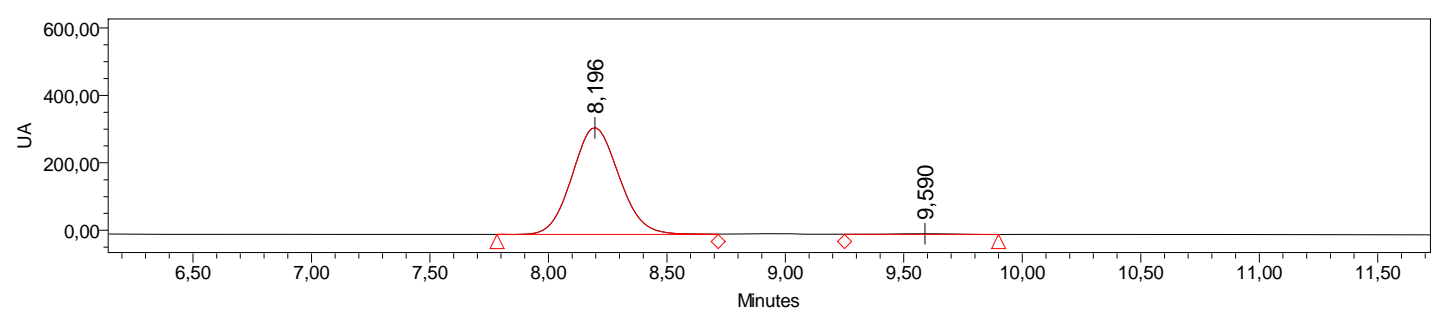


CHIRALPAK IA, 90:10 (hexane:iPrOH), $1.0 \mathrm{~mL} / \mathrm{min}, \lambda=210 \mathrm{~nm}$

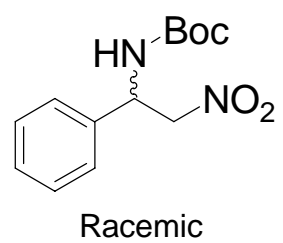

Processed Channel Descr.: 254 nm
\begin{tabular}{|c|l|c|c|r|r|}
\hline & $\begin{array}{c}\text { Processed } \\
\text { Channel Descr. }\end{array}$ & RT & Area & $\%$ Area & Height \\
\hline 1 & $254 \mathrm{~nm}$ & 12,187 & 117493 & 4,45 & 5582 \\
\hline 2 & $254 \mathrm{~nm}$ & 13,020 & 2523342 & 95,55 & 90369 \\
\hline
\end{tabular}
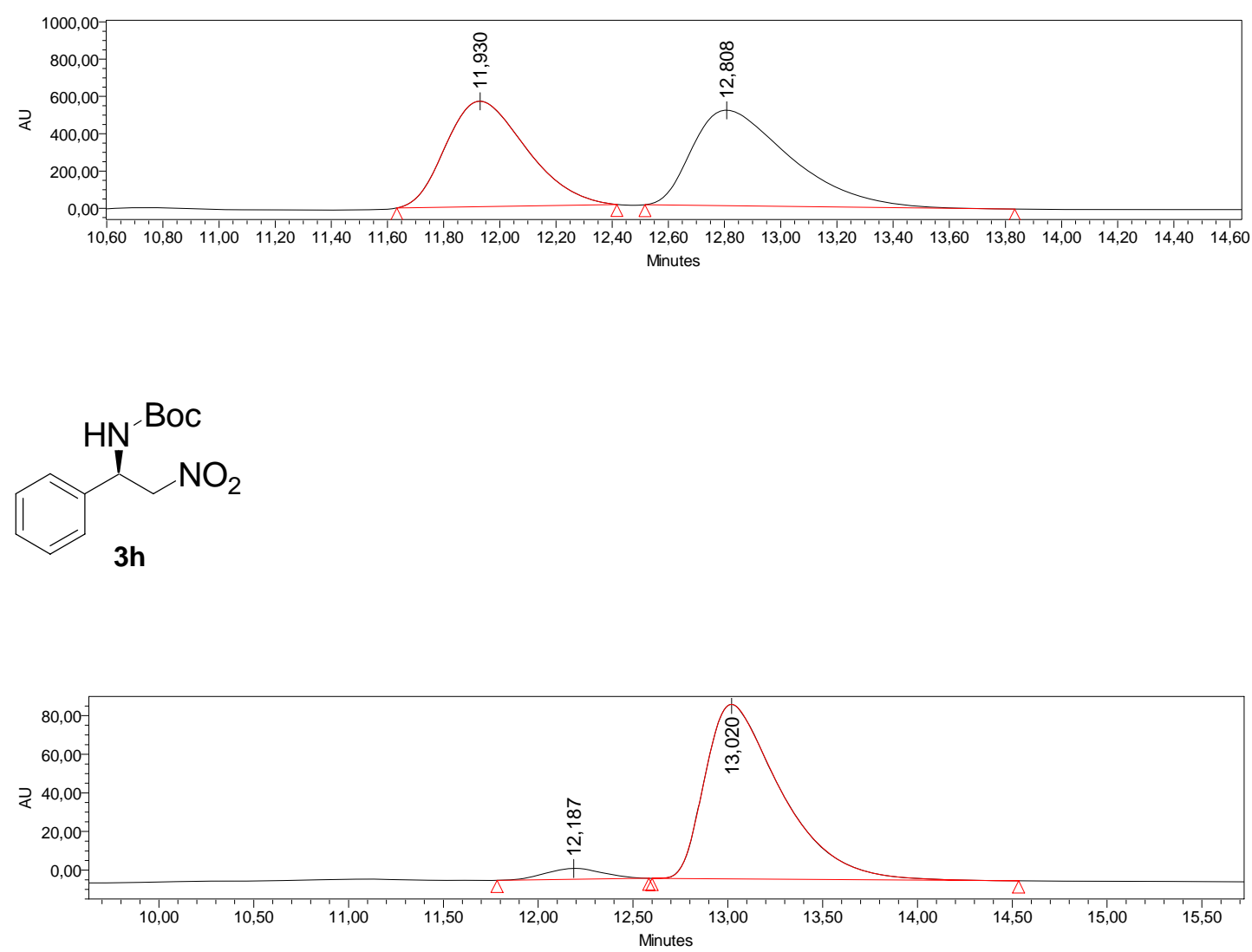
Chiralpak IA, hexane:iPrOH (90:10), $0.8 \mathrm{~mL} / \mathrm{min}, \lambda=210 \mathrm{~nm}$

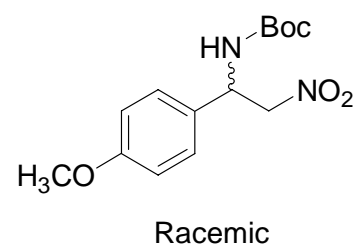

Processed Channel Descr.: @210nm

\begin{tabular}{|r|l|c|r|r|r|}
\hline & $\begin{array}{c}\text { Processed } \\
\text { Channel Descr. }\end{array}$ & RT & \multicolumn{1}{c|}{ Area } & \% Area & Height \\
\hline 1 & $@ 210 \mathrm{~nm}$ & 19,406 & 226380 & 4,32 & 6988 \\
\hline 2 & $@ 210 \mathrm{~nm}$ & 21,947 & 5019072 & 95,68 & 121154 \\
\hline
\end{tabular}

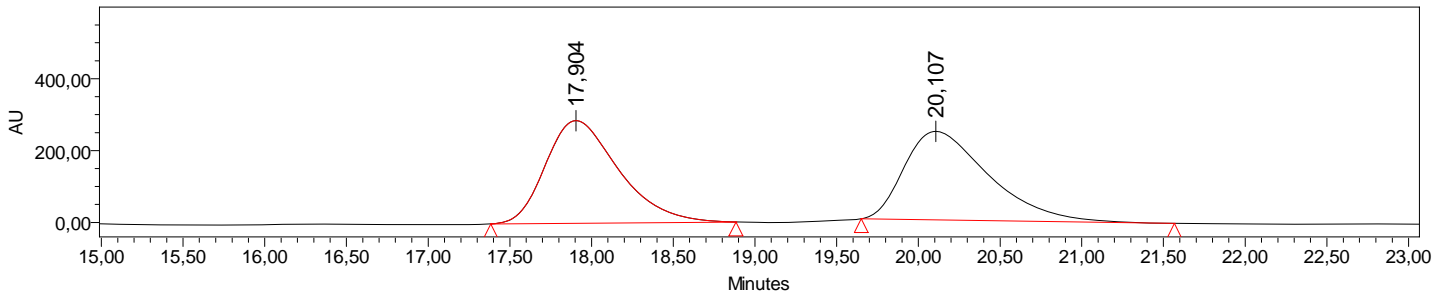

(NO)

$3 \mathbf{i}$

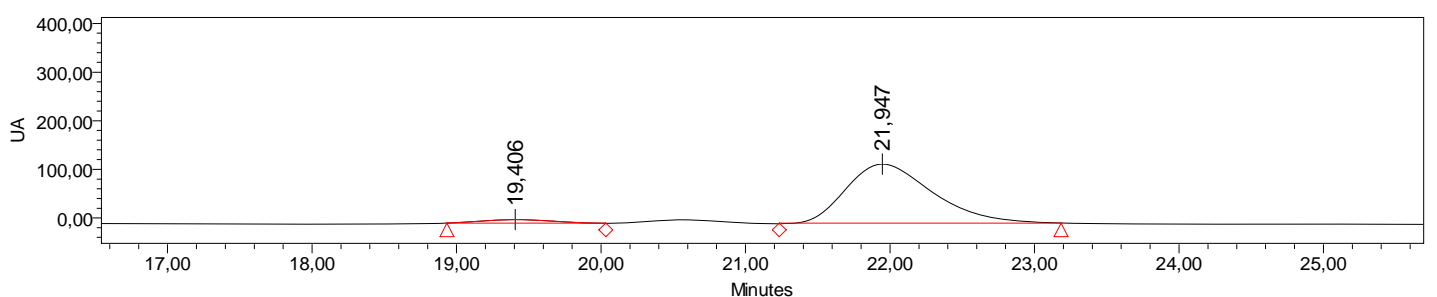


CHIRALPAK IA, 90:10 (hexane:iPrOH), $1.0 \mathrm{~mL} / \mathrm{min}, \lambda=220 \mathrm{~nm}$ (after crystallization $96 \%$ ee, before $80 \%$ ee)

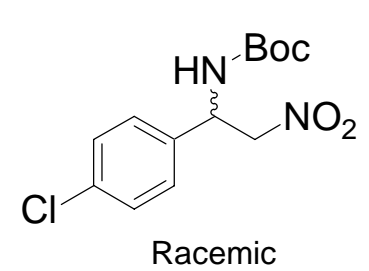

Processed Channel Descr.: 220

\begin{tabular}{|r|l|c|r|r|r|}
\hline & $\begin{array}{c}\text { Processed } \\
\text { Channel Descr. }\end{array}$ & RT & \multicolumn{1}{|c|}{ Area } & \% Area & Height \\
\hline 1 & 220 & 12,016 & 459775 & 1,84 & 22157 \\
\hline 2 & 220 & 15,993 & 24499766 & 98,16 & 759273 \\
\hline
\end{tabular}
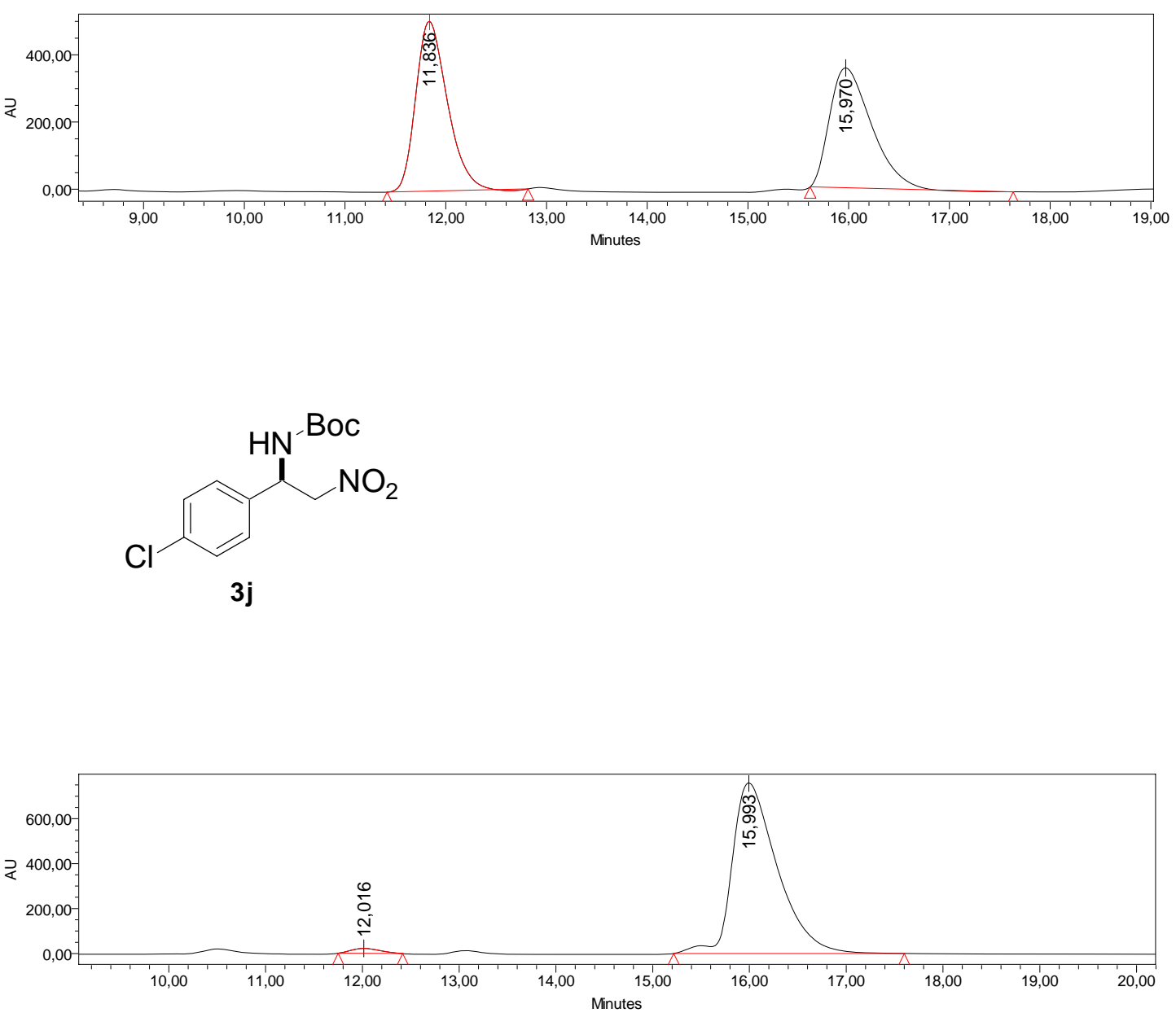
chiralpak IA 90:10 hex:iPrOH, 90:10, 1.0mL/min, $\lambda=210 \mathrm{~nm}$<smiles>CCOC(=O)NC(C[N+](=O)[O-])c1ccc(C(F)(F)F)cc1</smiles>
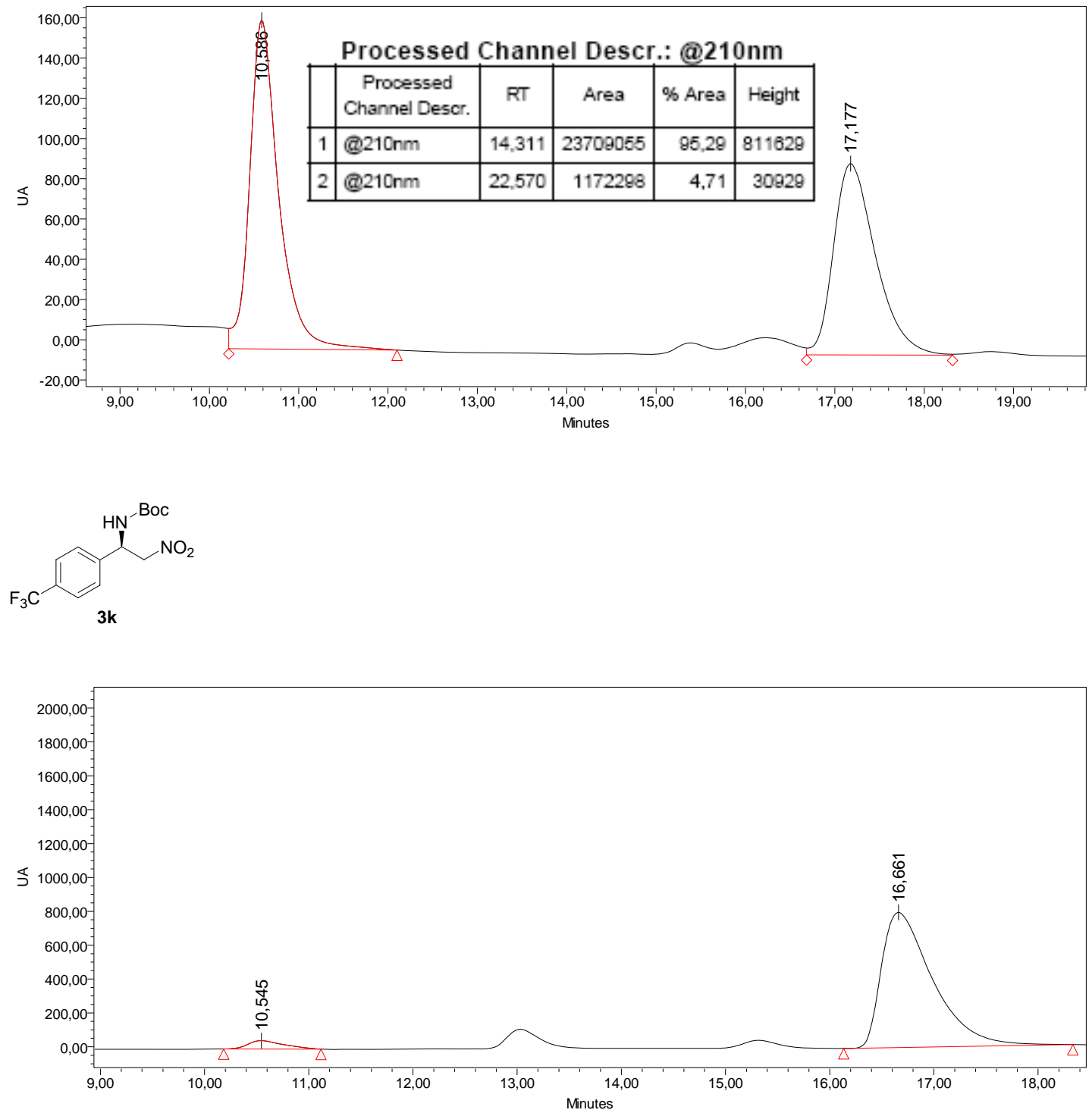
Chiralpak IA 90:10 hex : iPrOH, 90:10, 1.0mL/min, $\lambda=210 \mathrm{~nm}$
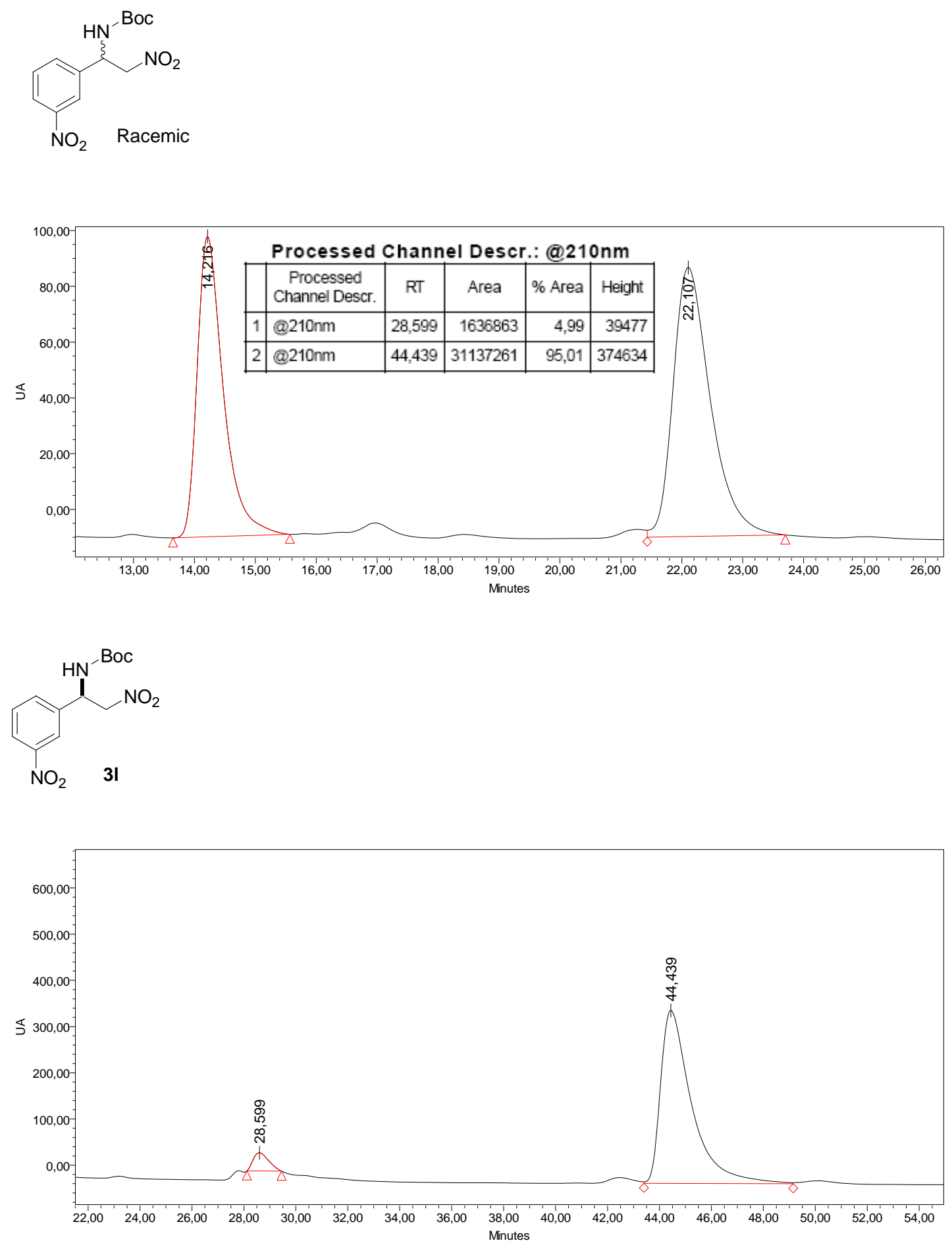
CHIRALPAK IA, 90:10 (hexane:iPrOH), $1.0 \mathrm{~mL} / \mathrm{min}, \lambda=220 \mathrm{~nm}$ (after crystalisation $94 \%$ ee, before crystalisation $90 \%$ )<smiles>CC(C)(C)OC(=O)N[C@H](C[N+](=O)[O-])c1cccc2ccccc12</smiles>

Processed Channel Descr.: 220

\begin{tabular}{|r|l|c|r|r|r|}
\hline & $\begin{array}{c}\text { Processed } \\
\text { Channel Descr. }\end{array}$ & RT & \multicolumn{1}{c|}{ Area } & $\%$ Area & Height \\
\hline 1 & 220 & 13,628 & 827103 & 2,91 & 35130 \\
\hline 2 & 220 & 19,422 & 27587152 & 97,09 & 812537 \\
\hline
\end{tabular}
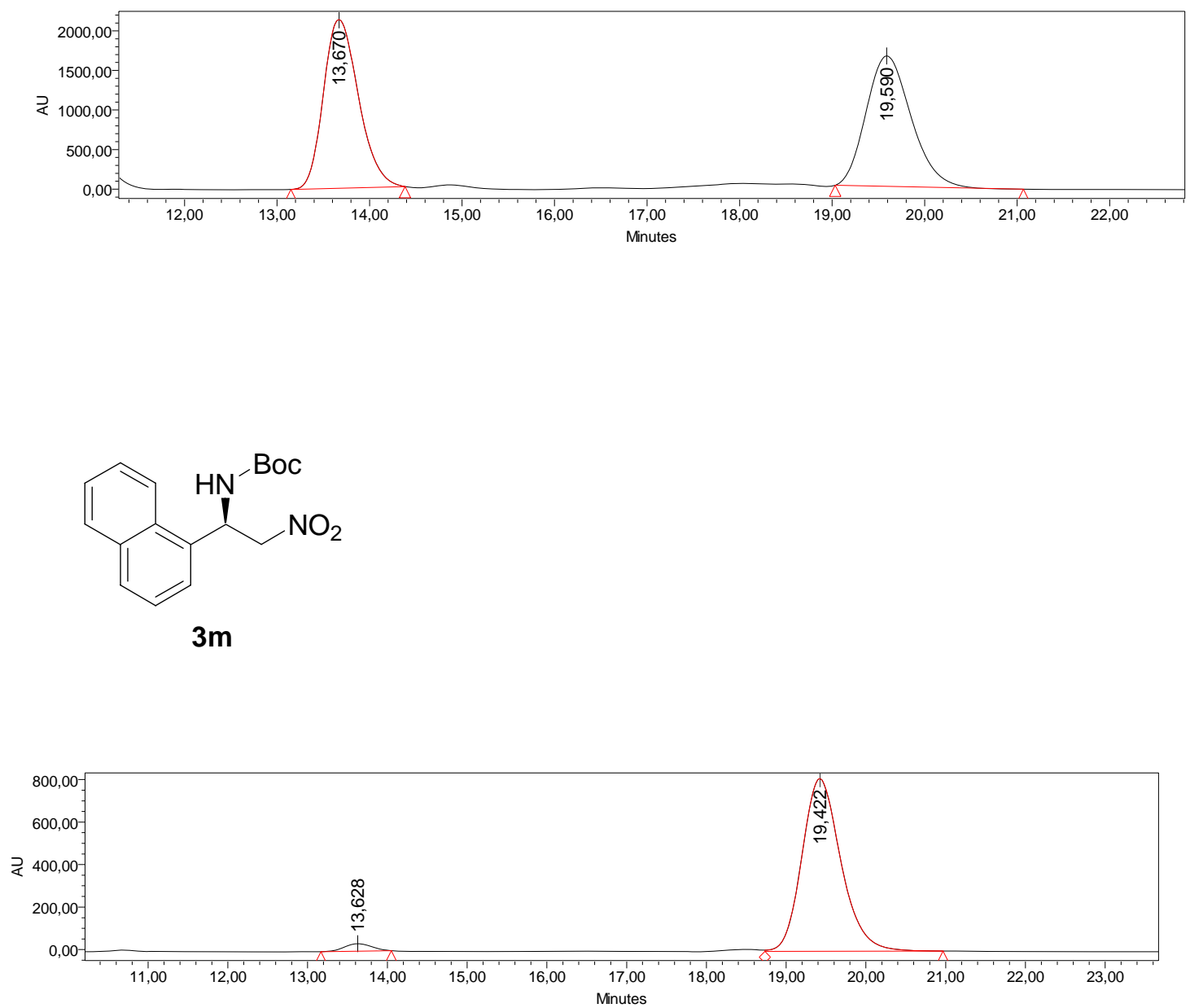

S29 
Chiralcel OD, 90:10 hex:iPrOH, $0.5 \mathrm{~mL} / \mathrm{min}, \lambda=210 \mathrm{~nm}$

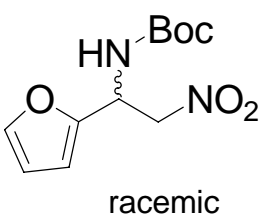

Processed Channel Descr.: @210nm
\begin{tabular}{|l|l|c|c|r|r|}
\hline & $\begin{array}{c}\text { Processed } \\
\text { Channel Descr. }\end{array}$ & RT & Area & $\%$ Area & Height \\
\hline 1 & $@ 210 \mathrm{~nm}$ & 18,379 & 4275035 & 8,11 & 76160 \\
\hline 2 & $@ 210 \mathrm{~nm}$ & 21,117 & 48407927 & 91,89 & 796982 \\
\hline
\end{tabular}
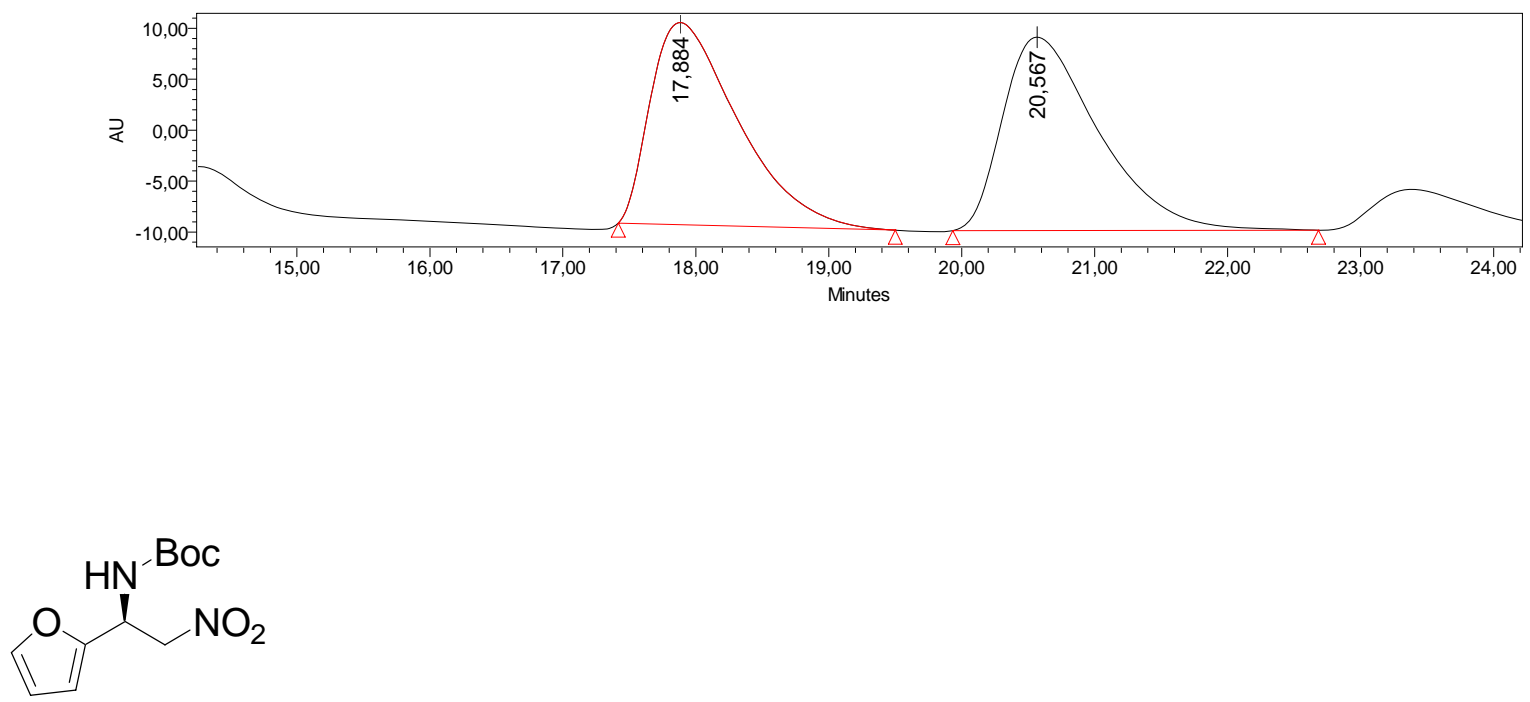

3n

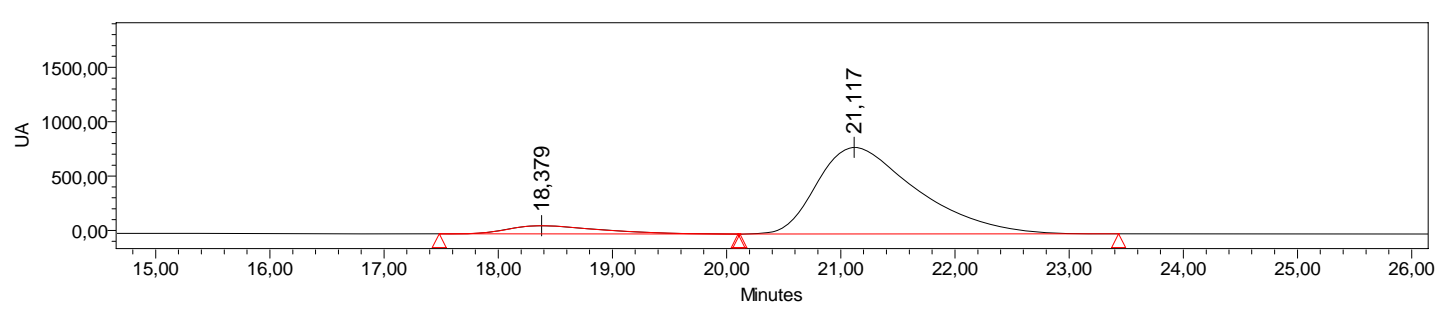


Chiralpak IA, 99:1 hex:iPrOH, $0.75 \mathrm{~mL} / \mathrm{min}, \lambda=210 \mathrm{~nm}^{11}$<smiles>CC(C)C(NC(CCc1ccccc1)C(=O)OC(C)(C)C)C(=O)[O-]</smiles>

racemic

Processed Channel Descr.: @210nm

\begin{tabular}{|r|l|c|r|r|r|}
\hline & $\begin{array}{c}\text { Processed } \\
\text { Channel Descr. }\end{array}$ & RT & \multicolumn{1}{c|}{ Area } & \% Area & Height \\
\hline 1 & $@ 210 \mathrm{~nm}$ & 20,808 & 6902430 & 10,21 & 155547 \\
\hline 2 & $@ 210 \mathrm{~nm}$ & 23,044 & 2605929 & 3,85 & 47233 \\
\hline 3 & $@ 210 \mathrm{~nm}$ & 26,183 & 80727 & 0,12 & 3217 \\
\hline 4 & $@ 210 \mathrm{~nm}$ & 27,823 & 58016644 & 85,82 & 916330 \\
\hline
\end{tabular}

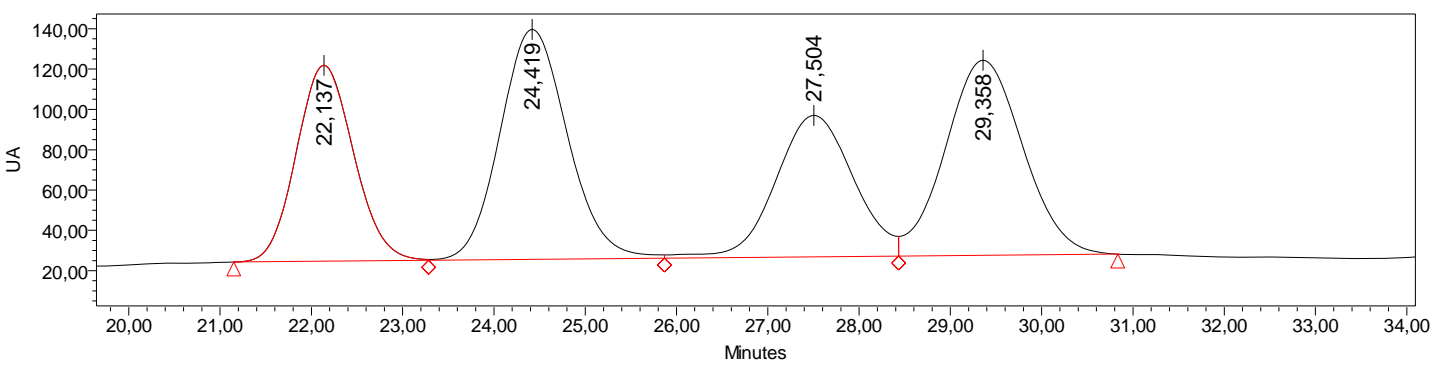

$\underbrace{\mathrm{NO}_{2}}_{\mathrm{Me}}$

$11 a$

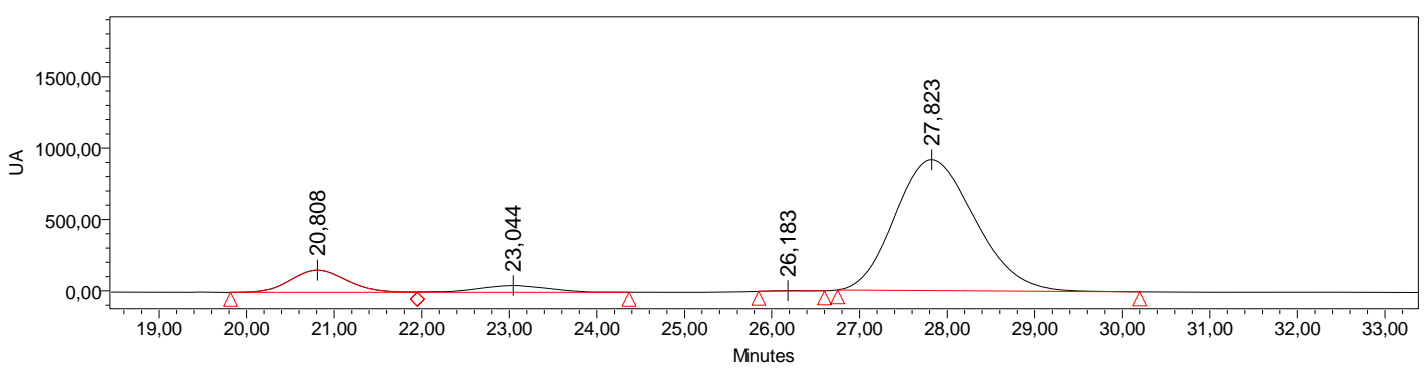


Conditions for the determination of isomers composition

Chiralpak IA, hex:iPrOH, 98:2, $0.5 \mathrm{~mL} / \mathrm{min}, \lambda=210 \mathrm{~nm}$<smiles>CC(C)(C)OC(=O)NC(c1ccccc1)C([N+](=O)[O-])[N+](=O)[O-]</smiles>

Processed Channel Descr.: @210nm

\begin{tabular}{|r|l|c|r|r|r|}
\hline & $\begin{array}{c}\text { Processed } \\
\text { Channel Descr. }\end{array}$ & RT & \multicolumn{1}{c|}{ Area } & \% Area & Height \\
\hline 1 & $@ 210 \mathrm{~nm}$ & 44,704 & 522044 & 2,93 & 7584 \\
\hline 2 & $@ 210 \mathrm{~nm}$ & 47,830 & 16026527 & 89,87 & 201472 \\
\hline 3 & $@ 210 \mathrm{~nm}$ & 56,249 & 963554 & 5,40 & 11052 \\
\hline 4 & $@ 210 \mathrm{~nm}$ & 75,426 & 320617 & 1,80 & 2856 \\
\hline
\end{tabular}

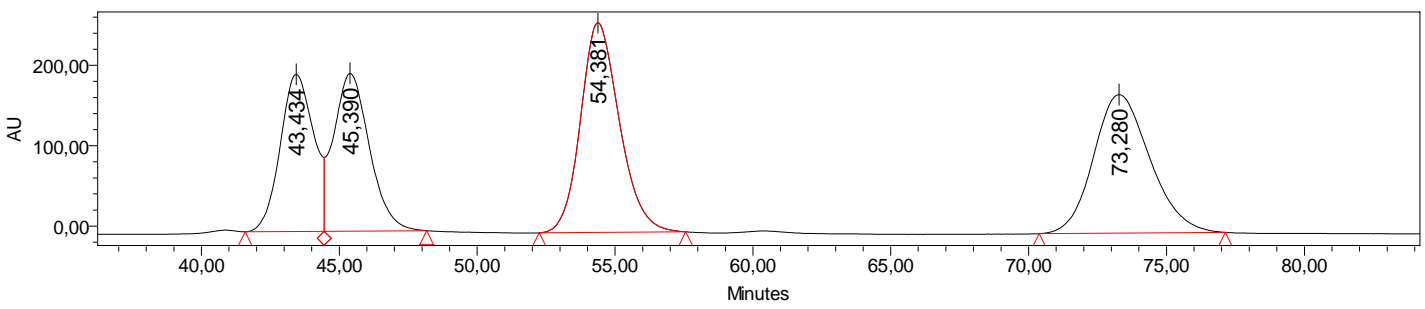<smiles>CC(C)(C)OC(=O)NC(c1ccccc1)C([N+](=O)[O-])[N+](=O)[O-]</smiles>

$11 \mathrm{~h}$

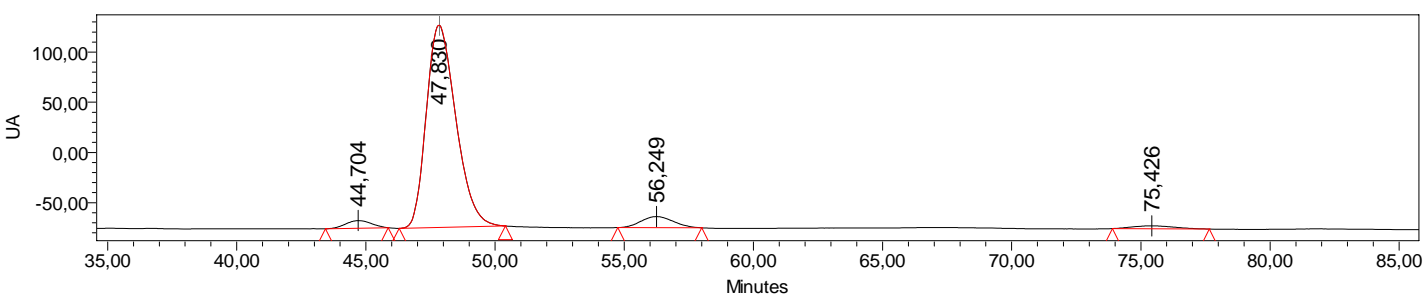

Conditions for the isomers identification by comparison with published values ${ }^{10}$

Chiralcel OJ, 99:1 hex:iPrOH, $1 \mathrm{~mL} / \mathrm{min}, \lambda=254 \mathrm{~nm}$

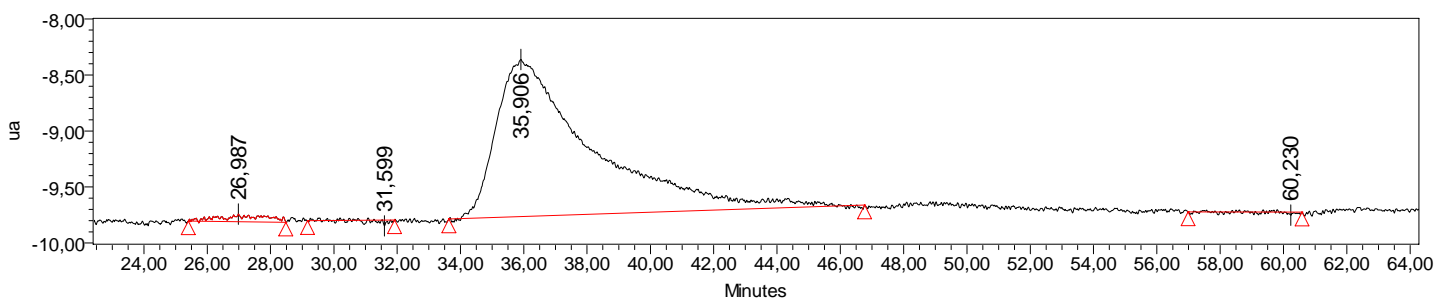

${ }^{10}$ Yoon, T. P.; Jacobsen, E. N. Angew. Chem. Int. Ed. 2005, 44, 466-468. 
Chiralpak AD, 92:8 hex:iPrOH, $1 \mathrm{~mL} / \mathrm{min}, \lambda=210 \mathrm{~nm}^{11}$<smiles>COc1ccc(C(NC(=O)OC(C)(C)C)C(C)[N+](=O)[O-])cc1</smiles>

$11 \mathrm{i}$

Processed Channel Descr.: @210nm

\begin{tabular}{|c|l|c|r|r|r|}
\hline & $\begin{array}{c}\text { Processed } \\
\text { Channel Descr. }\end{array}$ & RT & \multicolumn{1}{c|}{ Area } & $\%$ Area & Height \\
\hline 1 & $@ 210 \mathrm{~nm}$ & 18,044 & 291083 & 4,99 & 8830 \\
\hline 2 & $@ 210 \mathrm{~nm}$ & 20,555 & 5224551 & 89,53 & 136652 \\
\hline 3 & $@ 210 \mathrm{~nm}$ & 22,555 & 283401 & 4,86 & 7630 \\
\hline 4 & $@ 210 \mathrm{~nm}$ & 27,371 & 36799 & 0,63 & 1049 \\
\hline
\end{tabular}

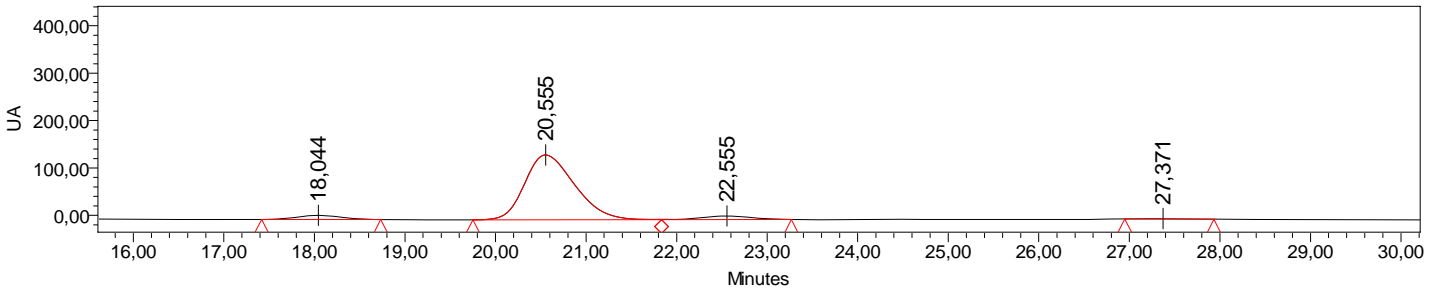

Chiralpak AD, 92:8 hex:iPrOH, $1 \mathrm{~mL} / \mathrm{min}, \lambda=210 \mathrm{~nm}^{11}$<smiles>COC(=O)N[C@@H](NC(C)c1ccc(Cl)cc1)C(C)[N+](=O)[O-]</smiles>

$11 \mathrm{j}$
Processed Channel Descr.: @210nm

\begin{tabular}{|r|l|c|r|r|r|}
\hline & $\begin{array}{c}\text { Processed } \\
\text { Channel Descr. }\end{array}$ & RT & \multicolumn{1}{c|}{ Area } & \% Area & Height \\
\hline 1 & $@ 210 \mathrm{~nm}$ & 15,385 & 112442 & 0,82 & 4271 \\
\hline 2 & $@ 210 \mathrm{~nm}$ & 18,123 & 1156104 & 8,45 & 33059 \\
\hline 3 & $@ 210 \mathrm{~nm}$ & 19,614 & 2144431 & 15,68 & 64660 \\
\hline 4 & $@ 210 \mathrm{~nm}$ & 21,104 & 10264984 & 75,05 & 171858 \\
\hline
\end{tabular}

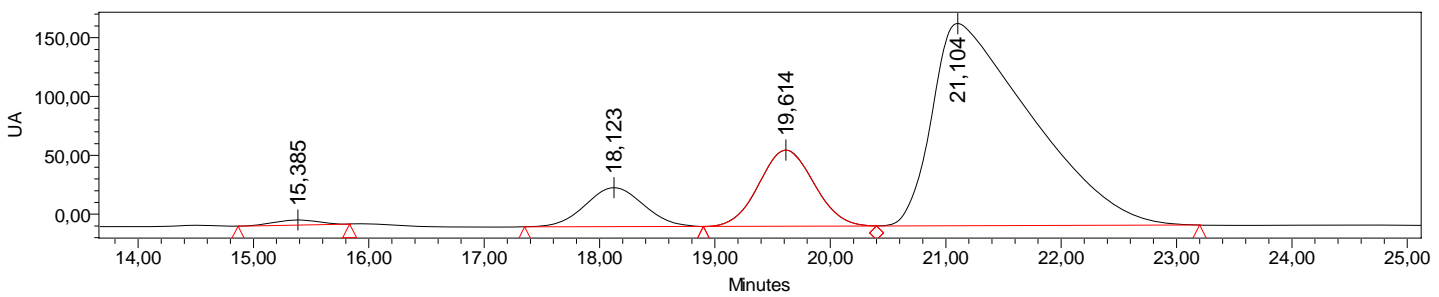

${ }^{11}$ Conditions reported in Yoon, T. P.; Jacobsen, E. N. Angew. Chem. Int. Ed. 2005, 44, 466-468. 